TITLE:

\title{
Wegner estimates, Lifshitz tails, and Anderson localization for Gaussian random magnetic fields
}

$\operatorname{AUTHOR}(\mathrm{S})$ :

Ueki, Naomasa

CITATION:

Ueki, Naomasa. Wegner estimates, Lifshitz tails, and Anderson localization for Gaussian random magnetic fields. Journal of Mathematical Physics 2016, 57(7): 071502.

ISSUE DATE:

2016-07-01

URL:

http://hdl.handle.net/2433/232506

\section{RIGHT:}

The following article appeared in 'Journal of Mathematical Physics 57 , 071502 (2016)' and may be found at

https://aip.scitation.org/doi/abs/10.1063/1.4959219.; The full-text file will be made open to the public on 01 July 2017 in accordance with publisher's 'Terms and Conditions for Self-Archiving'. 


\title{
Wegner estimates, Lifshitz tails, and Anderson localization for Gaussian random magnetic fields
}

\author{
Naomasa Uekia) \\ Graduate School of Human and Environmental Studies, Kyoto University, \\ Kyoto 606-8501, Japan
}

(Received 26 March 2015; accepted 10 July 2016; published online 25 July 2016)

The Wegner estimate for the Hamiltonian of the Anderson model for the special Gaussian random magnetic field is extended to more general magnetic fields. The Lifshitz tail upper bounds of the integrated density of states as analyzed by Nakamura are reviewed and extended so that Gaussian random magnetic fields can be treated. By these and multiscale analysis, the Anderson localization at low energies is proven. Published by AIP Publishing. [http://dx.doi.org/10.1063/1.4959219]

\section{INTRODUCTION}

For any $L \geq 1$ and $\omega$ in a probability space, we consider the self-adjoint operator

$$
H_{L}^{\omega}:=\sum_{\iota=1}^{2}\left(i \partial_{\iota}+A_{L, \iota}^{\omega}(x)\right)^{2}
$$

with the Dirichlet boundary condition on the open square $\Lambda_{L}=(-L / 2, L / 2)^{2}$ centered on the origin and with side length $L$; here $i=\sqrt{-1}$ and $A_{L}^{\omega}$ is a $C^{1}$-map from $\Lambda_{L}$ to $\mathbb{R}^{2}$ satisfying $\nabla \times A_{L}^{\omega}:=\partial_{1} A_{L, 2}^{\omega}-\partial_{2} A_{L, 1}^{\omega}=B^{\omega}$. Its spectrum depends only on $B^{\omega}$ and is independent of the choice of the vector potential $A_{L}^{\omega}$. This is the Schrödinger operator for the magnetic field $B^{\omega}$.

For $B^{\omega}$, we take a Gaussian random field on $\mathbb{R}^{2}$. We assume that $B^{\omega}(x)$ is stationary with respect to the shift in the space variable $x \in \mathbb{R}^{2}$ : the random fields $B^{\omega}(\cdot)$ and $B^{\omega}(x+\cdot)$ obey a similar law. Moreover, we assume that its covariance function $V(x-y)=\operatorname{Cov}\left(B^{\omega}(x), B^{\omega}(y)\right)$ is

$$
V(x)=\int \sigma(x-y) \sigma(y) d y,
$$

where $\sigma$ is a function satisfying the following conditions:

(A1) (i) $|\widehat{\sigma}(\xi)|^{2}(1+|\xi|)^{4} \in L^{1}\left(\mathbb{R}^{2}\right)$,

(ii) $\lim _{\mathcal{\varepsilon} \downarrow 0} \sup _{\mathcal{R} \in[1, \infty)}\left|\left\{\xi \in \mathbb{R}^{2}:|\xi| \leq \mathcal{R},|\widehat{\sigma}(\xi)|^{2}(1+|\xi|)^{m} \leq \varepsilon\right\}\right| /\left(\mathcal{R}^{2} \varepsilon^{\mu}\right)=0$ for some $\mu$ $\in(0, \infty)$ and $m \in(8, \infty)$,

where

$$
\widehat{f}(\xi)=\int_{\mathbb{R}^{2}} \exp (-2 \pi i \xi \cdot x) f(x) d x
$$

is the Fourier transform of an integrable function $f$.

(A2) $\exists \varepsilon_{1}, \varepsilon_{2}, c_{1}, \ldots, c_{5} \in(0, \infty), \theta_{*} \in \mathbb{R} /(2 \pi \mathbb{Z}), v>3 / 2$ such that

(i) $\sigma(x) \geq 0$ for any $x \in \mathbb{R}^{2}$,

(ii) $\operatorname{supp} \sigma$ is compact and $\partial \operatorname{supp} \sigma$ is a finite union of piecewise $C^{1}$ closed curves,

(iii) $\sigma(x) \leq c_{1} d(x, \partial \operatorname{supp} \sigma)^{v}$ for any $x \in \operatorname{supp} \sigma$,

(iv) $\forall s \in\left[0, \varepsilon_{1}\right]$ and $y \in\left\{y: 0<d(y, \operatorname{supp} \sigma) \leq \varepsilon_{1}\right\}, \mathcal{H}^{1}(\{x \in \operatorname{supp} \sigma: d(x, y)=s+$ $d(y, \operatorname{supp} \sigma)\}) \leq c_{2} s^{1 / 2}$,

\footnotetext{
a)Electronic mail: ueki@math.h.kyoto-u.ac.jp
} 
(v) $\forall s \in\left[0, \varepsilon_{1}\right]$ and $y \in \Sigma\left(\theta_{*}, \varepsilon_{1}, \varepsilon_{2}\right), \mathcal{H}^{1}\left(\left\{x: \sigma(x) \geq c_{3} s^{v}, d(x, y)=s+d(y, \operatorname{supp} \sigma)\right\}\right)$ $\geq c_{4} s^{1 / 2}$

(vi) $\mathcal{H}^{1}\left\{y \in \Sigma\left(\theta_{*}, \varepsilon_{1}, \varepsilon_{2}\right): d(y, \operatorname{supp} \sigma)=t\right\} \geq c_{5}$ for $\forall t \in\left[0, \varepsilon_{1}\right]$,

where $d(\cdot, \cdot)$ is the Euclidean distance, $\mathcal{H}^{1}$ is the one-dimensional Hausdorff measure, $\Sigma\left(\theta_{*}, \varepsilon_{1}, \varepsilon_{2}\right)=$ $\left\{y: 0<d(y, \operatorname{supp} \sigma) \leq \varepsilon_{1},\left|\arg y-\theta_{*}\right| \leq \varepsilon_{2}\right\}$ and $\arg y \in \mathbb{R}$ such that $y=|y|(\cos \arg y, \sin \arg y)$.

In this paper, we prove the following.

Theorem 1 (Wegner estimate). Under the above assumptions, there exist positive finite constants $C_{0}, C_{1}$, and $C_{2}$ such that

$$
\mathbb{E}\left[\operatorname{Tr}\left[\chi_{[E-\eta, E+\eta]}\left(H_{L}^{\omega}\right)\right]\right] \leq C_{0} R^{2} \eta L^{C_{1}}
$$

for any $R \in[1, \infty), L \geq \sqrt{R} \vee C_{2}$, and $E, \eta>0$ satisfying $E+\eta \leq R$.

By this theorem, the results regarding the Lifshitz tail owing to Nakamura ${ }^{12}$ and the theory on the multi-scale analysis by Germinet and Klein, ${ }^{8}$ we obtain the following.

Corollary (Anderson localization). Under the same assumptions in the last theorem, the operator

$$
H^{\omega}:=\sum_{\iota=1}^{2}\left(i \partial_{\iota}+A_{\iota}^{\omega}(x)\right)^{2}
$$

with a $C^{1}$ vector potential $A^{\omega}$ on $\mathbb{R}^{2}$ such that $\nabla \times A^{\omega}=B^{\omega}$ exhibits Anderson localization at low energies defined by the following: there exists a positive finite constant $\varepsilon_{0}$ such that $\left[0, \varepsilon_{0}\right]$ is included in the pure point spectrum of $H^{\omega}$, the corresponding eigenfunctions decay exponentially, and

$$
\mathbb{E}\left[\sup _{t}\left\||x|^{p} e^{-i t H^{\omega}} 1_{I}\left(H^{\omega}\right) 1_{K}\right\|_{L^{2}\left(\mathbb{R}^{2}\right) \rightarrow L^{2}\left(\mathbb{R}^{2}\right)}\right]<\infty
$$

for any $p \in(0, \infty), I \subset\left[0, \varepsilon_{0}\right]$, and any compact set $K$ in $\mathbb{R}^{2}$, where $\|\cdot\|_{L^{2}\left(\mathbb{R}^{2}\right) \rightarrow L^{2}\left(\mathbb{R}^{2}\right)}$ is the operator norm of bounded operators on $L^{2}\left(\mathbb{R}^{2}\right)$.

The above theorem and its corollary are generalizations of results in a previous paper ${ }^{19}$ for the following typical example:

$$
V(x)=\int \widetilde{\sigma}(x-y) \widetilde{\sigma}(y) d y,
$$

where

$$
\widetilde{\sigma}(x)=\mathcal{P}(\Delta)\left(\bar{\sigma}^{2}-|x|^{2}\right)_{+}^{v},
$$

$a_{+}=\max \{a, 0\}$ is the positive part, $\bar{\sigma} \in(0, \infty), v \in(3 / 2, \infty), \Delta=\partial_{1}^{2}+\partial_{2}^{2}$, and $\mathcal{P}$ is a non-zero polynomial of degree less than $(v-3 / 2) / 2$.

As discussed in the previous paper, ${ }^{19}$ this work is based on Erdôs and Hasler's Wegner estimate for special random magnetic fields: ${ }^{5-7}$ because the Schrödinger operator for random magnetic fields does not feature monotonicity used in the original work by Wegner's estimate ${ }^{20}$ Erdôs and Hasler made it possible to apply integration by parts twice under a nondegeneracy condition. The nondegeneracy condition is analogous to the nondegeneracy in the Malliavin sense used to prove the regularity of the probability density function of the solution of the stochastic differential equation of Itô type. The importance of the integration by parts on the probability space is also common in Malliavin calculus. Hence, we shall also use Malliavin calculus. However, we use a different representation of the Gaussian random field given by

$$
B^{\omega}(x)=B+\int\left(\operatorname{Re}(\widehat{\sigma}(\xi))\left(\begin{array}{c}
\cos (2 \pi x \xi) \\
-\sin (2 \pi x \xi)
\end{array}\right)+\operatorname{Im}(\widehat{\sigma}(\xi))\left(\begin{array}{c}
\sin (2 \pi x \xi) \\
\cos (2 \pi x \xi)
\end{array}\right)\right) \cdot \omega(d \xi),
$$

where $\omega={ }^{t}\left(\omega_{1}, \omega_{2}\right)$, and $\omega_{1}$ and $\omega_{2}$ are the independent copies of white noise on $\mathbb{R}^{2}$ (cf. Nualart ${ }^{13}$ ). This is the so-called spectral representation of stationary random fields. By this representation, 
we do not need the special form of Eq. (8). Then, our probability space is the Wiener space $\mathcal{D}^{\prime}\left(\mathbb{R}^{2}\right) \oplus \mathcal{D}^{\prime}\left(\mathbb{R}^{2}\right)$ with the Cameron-Martin space $L^{2}\left(\mathbb{R}^{2}\right) \oplus L^{2}\left(\mathbb{R}^{2}\right)$. The $\ell$ th eigenvalue $\lambda_{\ell}\left(H_{L}^{\omega}\right)$ is a smooth Wiener functional on this space and the following estimate of the $H$-derivative $D \lambda_{\ell}\left(H_{L}^{\omega}\right)$ is one of the key estimates in this paper (cf. Shigekawa ${ }^{16}$ ).

Lemma 1.1 (A bound of the gradient norm by a quadratic form). Under condition (A1), there exist universal constants $c_{1}, c_{2}, m_{1}, m_{2}, m_{3}, m_{4}, m_{5} \in(0, \infty)$ such that for any $L$ and $\omega$, one can choose $a x_{\omega} \in \Lambda_{L}$ so that for any $R \in(0, \infty)$, the following inequality holds whenever $\lambda_{\ell}\left(H_{L}^{\omega}\right)<R$ and $x_{0}$ satisfies $\left|x_{0}-x_{\omega}\right| \leq c_{2} L^{-m_{3}}\left(\left\|B^{\omega}\right\|_{W^{2,2}\left(\left(\Lambda_{L}\right)\right)}^{2}+R\right)^{-m_{4}}$,

$$
\begin{aligned}
& \left\|D \lambda_{\ell}\left(H_{L}^{\omega}\right)\right\|_{L^{2}\left(\mathbb{R}^{2}\right) \oplus L^{2}\left(\mathbb{R}^{2}\right)}^{2}
\end{aligned}
$$

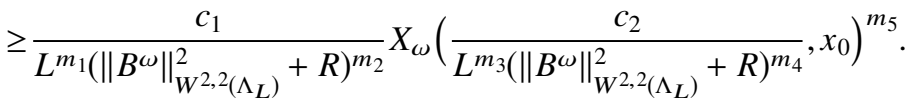

In this inequality, $X_{\omega}(\mathcal{R}, x)$ is the quadratic form in $\omega$ defined by

$$
X_{\omega}(\mathcal{R}, x)=\int_{0}^{\mathcal{R}}\left|\int_{|y| \leq r} B^{\omega}(x+y) d y\right|^{2} \frac{d r}{2 \pi r}
$$

for any $\mathcal{R}$ and $x \in \mathbb{R}^{2}$, and $\|\cdot\|_{W^{2,2}\left(\Lambda_{L}\right)}$ is the norm of the Sobolev space of functions on $\Lambda_{L}$ whose derivatives of order $\leq 2$ are square integrable.

This is essentially the estimate on nondegeneracy obtained by Erdôs and Hasler ${ }^{4}$ in their special case. We extended it to the special case Eq. (7) in the previous paper ${ }^{19}$ using the known information on the behavior of the Bessel function. In this paper, we extend it to the general setting of Eq. (2) using the spectral representation Eq. (9). Contrary to the case of Erdös and Hasler, the right-hand side of Eq. (10) still may attain zero. Therefore, we prepare the following as in the previous paper. ${ }^{19}$

Lemma 1.2 (Nondegeneracy of a quadratic form). Under condition (A2), there exists a finite positive constant $\overline{\mathcal{R}}$ such that, for any $p \in[1, \infty)$, there exists $c \in(0, \infty)$ satisfying

$$
\mathbb{E}\left[\frac{1}{X(\mathcal{R})^{p}}\right] \leq \frac{c}{\mathcal{R}^{p(2 v+5)(2 v+4) /(2 v+3)}}
$$

for any $\mathcal{R} \in(0, \overline{\mathcal{R}})$, where $X(\mathcal{R})=X_{\omega}(\mathcal{R}, 0)$.

This is a simple extension of the corollary of Lemma 4.1 in the previous paper. ${ }^{19}$ As examples satisfying both conditions (A1) and (A2), we have the following.

Example 1.1. In the representation Eq. (2), we can take $\sigma$ as follows:

(i) (An elliptic case) $\sigma(x)=\left(\bar{\sigma}^{2}-\sum_{j, k=1}^{2} a_{j k}\left(x-x^{0}\right)_{j}\left(x-x^{0}\right)_{k}\right)_{+}^{v}$, where $v \in(3 / 2, \infty),\left(a_{j k}\right)$ is a positive symmetric matrix, $x^{0}$ is a point in $\mathbb{R}^{2}$, and $a_{+}:=a \vee 0$ is the positive part for any $a \in \mathbb{R}$.

(ii) (A case where the variables are separated) $\sigma(x)=\sigma_{1}\left(\left(x-x^{0}\right) \cdot v_{1}\right) \sigma_{2}\left(\left(x-x^{0}\right) \cdot v_{2}\right)$, where $\left\{v_{1}, v_{2}\right\}$ is an orthonormal base of $\mathbb{R}^{2}, x^{0}$ is a point in $\mathbb{R}^{2}$, and $\sigma_{j}$ are the functions on $\mathbb{R}$ of the form $\sigma_{j}(t)=|t|^{v_{j}}\left(\overline{\sigma_{j}}-|t|\right)_{+}^{v_{j}}$ or $\sigma_{j}(t)=\left({\overline{\sigma_{j}}}^{2}-t^{2}\right)_{+}^{v_{j}}$ with some $\overline{\sigma_{j}}, v_{j} \in(0, \infty)$.

(iii) We can also construct such functions $\sigma$ by repeating appropriately taking either the convolution or the sum with functions of the form in (i) or (ii) above.

To prove that these examples satisfy condition (A1), we use the uniform nondegeneracy of the Bessel function proven in Appendix A. Appendix B is devoted to the proof of the fact that the example in (i) satisfies condition (A2). This condition is easily checked for the example in (ii).

The organization of this paper is as follows. Sections II-IV are devoted to the proof of the Wegner estimate: in Sections II and III, we prove Lemmas 1.1 and 1.2, respectively. In Section IV, we use these lemmas to prove Theorem 1. In Section V, we proceed to prove the Lifshitz tail for the corollary of Theorem 1. In Appendices A and B, we prove results used in the above explanation for Example 1.1. 


\section{PROOF OF LEMMA 1.1}

As in the previous paper, ${ }^{19}$ we take the vector potential as

$$
A_{L, 1}^{\omega}(x)=\left(\partial_{2} F_{L}^{\omega}\right)(x) \text { and } A_{L, 2}^{\omega}(x)=-\left(\partial_{1} F_{L}^{\omega}\right)(x)
$$

on $\Lambda_{L}$, where

$$
F_{L}^{\omega}(x)=\sum_{\boldsymbol{n} \in \mathbb{N}^{2}} \frac{\Phi_{\boldsymbol{n}, L}(x)}{E_{\boldsymbol{n}, L}} \int_{\Lambda_{L}} \Phi_{\boldsymbol{n}, L}(y) B^{\omega}(y) d y
$$

and

$$
E_{\boldsymbol{n}, L}=\left(\frac{\pi|\boldsymbol{n}|}{L}\right)^{2} \text { and } \Phi_{\boldsymbol{n}, L}(x)=\frac{2}{L} \prod_{\iota=1}^{2} \sin \left(n_{\iota} \pi\left(\frac{x_{\iota}}{L}+\frac{1}{2}\right)\right)
$$

for $\boldsymbol{n}=\left(n_{1}, n_{2}\right) \in \mathbb{N}^{2} .\left\{E_{\boldsymbol{n}, L}, \Phi_{\boldsymbol{n}, L}\right\}_{\boldsymbol{n} \in \mathbb{N}^{2}}$ are the eigenvalues and a complete orthonormal basis consisting of the eigenfunctions of the negative Dirichlet Laplacian $-\Delta_{\Lambda_{L}}^{D}$ (cf. Reed and Simon, ${ }^{15}$ p. 266). We take also the current $j_{L}^{\omega}(x)=\left(j_{1, L}^{\omega}(x), j_{2, L}^{\omega}(x)\right)$ of the normalized eigenfunction $\psi_{\ell}$ of the eigenvalue $\lambda_{\ell}\left(H_{L}^{\omega}\right)$ defined by

$$
j_{\iota, L}^{\omega}(x)=2 \operatorname{Re} \overline{\psi_{\ell}}\left(i \partial_{\iota}+A_{L, \iota}^{\omega}(x)\right) \psi_{\ell} .
$$

Then, by the Feynman-Hellmann theorem, we have

$$
\begin{gathered}
\left(D \lambda_{\ell}\left(H_{L}^{\omega}\right), \boldsymbol{\Phi}\right)_{L^{2}\left(\mathbb{R}^{2}\right) \oplus L^{2}\left(\mathbb{R}^{2}\right)}=\int_{\Lambda_{L}} j_{L}^{\omega}(x) \cdot\left(D A_{L}^{\omega}(x), \boldsymbol{\Phi}\right)_{L^{2}\left(\mathbb{R}^{2}\right) \oplus L^{2}\left(\mathbb{R}^{2}\right)} d x \\
\left(D A_{L, 1}^{\omega}(x), \boldsymbol{\Phi}\right)_{L^{2}\left(\mathbb{R}^{2}\right) \oplus L^{2}\left(\mathbb{R}^{2}\right)}=\sum_{\boldsymbol{n} \in \mathbb{N}^{2}} \frac{\left(\partial_{2} \Phi_{\boldsymbol{n}, L}\right)(x)}{E_{\boldsymbol{n}, L}} \int_{\Lambda_{L}} d y \Phi_{\boldsymbol{n}, L}(y) \int_{\mathbb{R}^{2}}\left(\operatorname{Re}(\widehat{\sigma}(\xi))\left(\begin{array}{c}
\cos (2 \pi y \xi) \\
-\sin (2 \pi y \xi)
\end{array}\right)\right. \\
+\operatorname{Im}(\widehat{\sigma}(\xi))\left(\begin{array}{c}
\sin (2 \pi y \xi) \\
\cos (2 \pi y \xi)
\end{array}\right) \cdot \boldsymbol{\Phi}(\xi) d \xi
\end{gathered}
$$

and

$$
\begin{aligned}
\left(D A_{L, 2}^{\omega}(x), \boldsymbol{\Phi}\right)_{L^{2}\left(\mathbb{R}^{2}\right) \oplus L^{2}\left(\mathbb{R}^{2}\right)}= & -\sum_{\boldsymbol{n} \in \mathbb{N}^{2}} \frac{\partial_{1} \Phi_{\boldsymbol{n}, L}(x)}{E_{\boldsymbol{n}, L}} \int_{\Lambda_{L}} d y \Phi_{\boldsymbol{n}, L}(y) \int_{\mathbb{R}^{2}}\left(\operatorname{Re}(\widehat{\sigma}(\xi))\left(\begin{array}{c}
\cos (2 \pi y \xi) \\
-\sin (2 \pi y \xi)
\end{array}\right)\right. \\
& \left.+\operatorname{Im}(\widehat{\sigma}(\xi))\left(\begin{array}{c}
\sin (2 \pi y \xi) \\
\cos (2 \pi y \xi)
\end{array}\right)\right) \cdot \boldsymbol{\Phi}(\xi) d \xi
\end{aligned}
$$

for any $\Phi \in L^{2}\left(\mathbb{R}^{2}\right) \oplus L^{2}\left(\mathbb{R}^{2}\right)$. Thus, we have

$$
\begin{aligned}
\left(D \lambda_{\ell}\left(H_{L}^{\omega}\right), \boldsymbol{\Phi}\right)_{L^{2}\left(\mathbb{R}^{2}\right) \oplus L^{2}\left(\mathbb{R}^{2}\right)}= & \int_{\mathbb{R}^{2}} d \xi \boldsymbol{\Phi}(\xi) \cdot \int_{\Lambda_{L}} d y\left(\operatorname{Re}(\widehat{\sigma}(\xi))\left(\begin{array}{c}
\cos (2 \pi y \xi) \\
-\sin (2 \pi y \xi)
\end{array}\right)\right. \\
& \left.+\operatorname{Im}(\widehat{\sigma}(\xi))\left(\begin{array}{c}
\sin (2 \pi y \xi) \\
\cos (2 \pi y \xi)
\end{array}\right)\right) \sum_{\boldsymbol{n} \in \mathbb{N}^{2}} \frac{\Phi_{\boldsymbol{n}, L}(y)}{E_{\boldsymbol{n}, L}}\left(\Phi_{\boldsymbol{n}, L}, \nabla \times j_{L}^{\omega}\right)_{L^{2}\left(\Lambda_{L}\right)},
\end{aligned}
$$


where $\nabla \times j_{L}^{\omega}=\partial_{1} j_{2, L}^{\omega}-\partial_{2} j_{1, L}^{\omega}$. Subsequently,

$$
\left\|D \lambda_{\ell}\left(H_{L}^{\omega}\right)\right\|_{L^{2}\left(\mathbb{R}^{2}\right) \oplus L^{2}\left(\mathbb{R}^{2}\right)}^{2}=\int_{\mathbb{R}^{2}} d \xi \widehat{\beta}(\xi)\left|\widehat{J}_{L}^{\omega}(\xi)\right|^{2},
$$

where $\widehat{\beta}(\xi):=|\widehat{\sigma}(\xi)|^{2}$ and

$$
J_{L}^{\omega}(y)=\sum_{\boldsymbol{n} \in \mathbb{N}^{2}} \frac{\Phi_{\boldsymbol{n}, L}(y)}{E_{\boldsymbol{n}, L}}\left(\Phi_{\boldsymbol{n}, L}, \nabla \times j_{L}^{\omega}\right)_{L^{2}\left(\Lambda_{L}\right)} .
$$

We now take $\mathcal{R} \in[1, \infty)$ and $\varepsilon \in(0,1)$ - to be specified later-to obtain the estimate

$\left\|D \lambda_{\ell}\left(H_{L}^{\omega}\right)\right\|_{L^{2}\left(\mathbb{R}^{2}\right) \oplus L^{2}\left(\mathbb{R}^{2}\right)}^{2} \geq \int_{|\xi| \leq \mathcal{R}, \widetilde{\beta}(\xi) \geq \varepsilon} d \xi \widehat{\beta}(\xi)\left|\widehat{J}_{L}^{\omega}(\xi)\right|^{2} \geq \frac{\varepsilon}{(2 \mathcal{R})^{m}} \int_{|\xi| \leq \mathcal{R}, \widetilde{\beta}(\xi) \geq \varepsilon} d \xi\left|\widehat{J}_{L}^{\omega}(\xi)\right|^{2}$,

where $\widetilde{\beta}(\xi)=\widehat{\beta}(\xi)(1+|\xi|)^{m}$ and $m$ is the number in condition (A1) (ii). We divide the right-hand side as

$$
\int_{|\xi| \leq \mathcal{R}, \widetilde{\beta}(\xi) \geq \varepsilon} d \xi\left|\widehat{J}_{L}^{\omega}(\xi)\right|^{2}=\left\|\widehat{J}_{L}^{\omega}(\xi)\right\|_{L^{2}\left(\mathbb{R}^{2}\right)}^{2}-\int_{|\xi| \geq \mathcal{R}} d \xi\left|\widehat{J}_{L}^{\omega}(\xi)\right|^{2}-\int_{|\xi| \leq \mathcal{R}, \widetilde{\beta}(\xi)<\varepsilon} d \xi\left|\widehat{J}_{L}^{\omega}(\xi)\right|^{2} .
$$

The first term is $\left\|J_{L}^{\omega}\right\|_{L^{2}\left(\Lambda_{L}\right)}^{2}$. By (3.1) in the previous paper, ${ }^{19}$ the second term is estimated as

$$
\int_{|\xi| \geq \mathcal{R}} d \xi\left|\widehat{J}_{L}^{\omega}(\xi)\right|^{2} \leq \frac{1}{\mathcal{R}^{4}} \int_{|\xi| \geq \mathcal{R}} d \xi|\xi|^{4}\left|\widehat{J}_{L}^{\omega}(\xi)\right|^{2} \leq \frac{\left\|\nabla \times j_{L}^{\omega}\right\|^{2}}{(2 \pi \mathcal{R})^{4}} \leq \frac{c_{1} L^{18}}{\mathcal{R}^{4}}\left(\left\|B^{\omega}\right\|_{W^{2,2}\left(\Lambda_{L}\right)}^{2}+R\right)^{4} .
$$

In this inequality and in the following, $\left\{c_{j}\right\}_{j=1,2, \ldots}$ are the universal constants. By condition (A1) (ii), for any $\eta \in(0,1)$, there exists $\varepsilon_{\eta} \in(0,1)$ such that

$$
\left|\left\{\xi \in \mathbb{R}^{2}:|\xi| \leq \mathcal{R}, \widetilde{\beta}(\xi) \leq \varepsilon\right\}\right| \leq \eta \mathcal{R}^{2} \varepsilon^{\mu}
$$

for any $\varepsilon \in\left(0, \varepsilon_{\eta}\right)$ and $\mathcal{R} \in[1, \infty)$. Then, the third term is estimated as

$$
\int_{|\xi| \leq \mathcal{R}, \widetilde{\beta}(\xi)<\varepsilon} d \xi\left|\widehat{J}_{L}^{\omega}(\xi)\right|^{2} \leq \eta \mathcal{R}^{2} \varepsilon^{\mu} \sup _{\xi}\left|\widehat{J}_{L}^{\omega}(\xi)\right|^{2}
$$

By $E_{n, L} \geq(\pi / L)^{2}$ and (3.1) in the previous paper, ${ }^{19}$ we have

$$
\begin{aligned}
& \sup _{\xi}\left|\widehat{J}_{L}^{\omega}(\xi)\right|^{2} \leq\left\|J_{L}^{\omega}\right\|_{L^{1}\left(\Lambda_{L}\right)}^{2} \leq L^{2}\left\|J_{L}^{\omega}\right\|_{L^{2}\left(\Lambda_{L}\right)}^{2} \\
& \leq \frac{L^{6}}{\pi^{4}}\left\|\nabla \times j_{L}^{\omega}\right\|_{L^{2}\left(\Lambda_{L}\right)}^{2} \leq c_{2} L^{24}\left(\left\|B^{\omega}\right\|_{W^{2,2}\left(\Lambda_{L}\right)}^{2}+R\right)^{4} .
\end{aligned}
$$

Thus we have

$$
\begin{aligned}
& \int_{|\xi| \leq \mathcal{R}, \widetilde{\beta}(\xi) \geq \varepsilon} d \xi\left|\widehat{J}_{L}^{\omega}(\xi)\right|^{2} \\
\geq & \left\|J_{L}^{\omega}\right\|_{L^{2}\left(\Lambda_{L}\right)}^{2}-\frac{c_{1} L^{18}}{\mathcal{R}^{4}}\left(\left\|B^{\omega}\right\|_{W^{2,2}\left(\Lambda_{L}\right)}^{2}+R\right)^{4}-c_{2} \eta \mathcal{R}^{2} \varepsilon^{\mu} L^{24}\left(\left\|B^{\omega}\right\|_{W^{2,2}\left(\Lambda_{L}\right)}^{2}+R\right)^{4} .
\end{aligned}
$$

We here take $\mathcal{R}=\left(2 c_{1}\right)^{1 / 4} L^{9 / 2}\left(\left\|B^{\omega}\right\|_{W^{2,2}\left(\Lambda_{L}\right)}^{2}+R\right)\left\|J_{L}^{\omega}\right\|_{L^{2}\left(\Lambda_{L}\right)}^{-1 / 2}$ so that the second term becomes $\left\|J_{L}^{\omega}\right\|_{L^{2}\left(\Lambda_{L}\right)}^{2} / 2$. By going back to the estimate Eq. (30), we have

$$
\begin{aligned}
& \left\|D \lambda_{\ell}\left(H_{L}^{\omega}\right)\right\|_{L^{2}\left(\mathbb{R}^{2}\right) \oplus L^{2}\left(\mathbb{R}^{2}\right)}^{2} \\
\geq & \frac{c_{3} \varepsilon\left\|J_{L}^{\omega}\right\|_{L^{2}\left(\Lambda_{L}\right)}^{m / 2}}{L^{9 m / 2}\left(\left\|B^{\omega}\right\|_{W^{2,2}\left(\Lambda_{L}\right)}^{2}+R\right)^{m}}\left\{\left\|J_{L}^{\omega}\right\|_{L^{2}\left(\Lambda_{L}\right)}^{2}-\frac{c_{4} \eta \varepsilon^{\mu} L^{33}}{\left\|J_{L}^{\omega}\right\|_{L^{2}\left(\Lambda_{L}\right)}}\left(\left\|B^{\omega}\right\|_{W^{2,2}\left(\Lambda_{L}\right)}^{2}+R\right)^{6}\right\} .
\end{aligned}
$$

We here take

$$
\varepsilon=\left\{\frac{\left\|J_{L}^{\omega}\right\|_{L^{2}\left(\Lambda_{L}\right)}^{3}}{2 c_{4} \eta L^{33}\left(\left\|B^{\omega}\right\|_{W^{2,2}\left(\Lambda_{L}\right)}^{2}+R\right)^{6}}\right\}^{1 / \mu}
$$


so that the terms in the last bracket become $\left\|J_{L}^{\omega}\right\|_{L^{2}\left(\Lambda_{L}\right)}^{2} / 2$. We here note that $\varepsilon<\varepsilon_{\eta}$ is satisfied for large $L$ by (3.1) in the previous paper. ${ }^{19}$ Then, we have

$$
\left\|D \lambda_{\ell}\left(H_{L}^{\omega}\right)\right\|_{L^{2}\left(\mathbb{R}^{2}\right) \oplus L^{2}\left(\mathbb{R}^{2}\right)}^{2} \geq \frac{c_{5}\left\|J_{L}^{\omega}\right\|_{L^{2}\left(\Lambda_{L}\right)}^{m / 2+3 / \mu}}{\eta^{1 / \mu} L^{9 m / 2+33 / \mu}\left(\left\|B^{\omega}\right\|_{W^{2,2}\left(\Lambda_{L}\right)}^{2}+R\right)^{m+6 / \mu}} .
$$

We next introduce $\mathcal{L} \in(1, \infty)$ to obtain the estimate

$$
\begin{aligned}
& \left\|J_{L}^{\omega}\right\|_{L^{2}\left(\Lambda_{L}\right)}^{2}=\sum_{\boldsymbol{n} \in \mathbb{N}^{2}}\left(\frac{L}{\pi|\boldsymbol{n}|}\right)^{4}\left(\Phi_{\boldsymbol{n}, L}, \nabla \times j_{L}^{\omega}\right)_{L^{2}\left(\Lambda_{L}\right)}^{2} \\
& \geq\left(\frac{L}{\pi \mathcal{L}}\right)^{4} \sum_{|\boldsymbol{n}| \leq \mathcal{L}}\left(\Phi_{\boldsymbol{n}, L}, \nabla \times j_{L}^{\omega}\right)_{L^{2}\left(\Lambda_{L}\right)}^{2}=\left(\frac{L}{\pi \mathcal{L}}\right)^{4}\left(\left\|\nabla \times j_{L}^{\omega}\right\|_{L^{2}\left(\Lambda_{L}\right)}^{2}-\sum_{|\boldsymbol{n}|>\mathcal{L}}\left(\Phi_{\boldsymbol{n}, L}, \nabla \times j_{L}^{\omega}\right)_{L^{2}\left(\Lambda_{L}\right)}^{2}\right) .
\end{aligned}
$$

As in Section 2 of the previous paper, ${ }^{19}$ we have

$$
\sum_{|\boldsymbol{n}|>\mathcal{L}}\left(\Phi_{\boldsymbol{n}, L}, \nabla \times j_{L}^{\omega}\right)_{L^{2}\left(\Lambda_{L}\right)}^{2} \leq c_{6} \frac{L^{24}}{\mathcal{L}^{2}}\left(\left\|B^{\omega}\right\|_{W^{2,2}\left(\Lambda_{L}\right)}^{2}+R\right)^{6} .
$$

By taking $\mathcal{L}$ so that the right-hand side becomes $\left\|\nabla \times j_{L}^{\omega}\right\|_{L^{2}\left(\Lambda_{L}\right)}^{2} / 2$ and using $\left\|\nabla \times j_{L}^{\omega}\right\|_{L^{2}\left(\Lambda_{L}\right)}=$ $\left\|\nabla j_{L}^{\omega}\right\|_{L^{2}\left(\Lambda_{L}\right)} \geq(\pi / L)\left\|j_{L}^{\omega}\right\|_{L^{2}\left(\Lambda_{L}\right)}$, we have

$$
\left\|J_{L}^{\omega}\right\|_{L^{2}\left(\Lambda_{L}\right)}^{2} \geq \frac{c_{7}\left\|\nabla \times j_{L}^{\omega}\right\|_{L^{2}\left(\Lambda_{L}\right)}^{6}}{L^{44}\left(\left\|B^{\omega}\right\|_{W^{2,2}\left(\Lambda_{L}\right)}^{2}+R\right)^{12}} \geq \frac{c_{8}\left\|j_{L}^{\omega}\right\|_{L^{2}\left(\Lambda_{L}\right)}^{6}}{L^{50}\left(\left\|B^{\omega}\right\|_{W^{2,2}\left(\Lambda_{L}\right)}^{2}+R\right)^{12}} .
$$

Finally, we use Lemma 3.2 as in Section 2 of the previous paper ${ }^{19}$ to complete the proof. For this proof, condition (A1) is not used.

\section{PROOF OF LEMMA 1.2}

As in Section 4 of the previous paper, ${ }^{19}$ we have only to prove as follows:

Lemma 3.1. Under condition (A2), there exist $\bar{R}, c \in(0, \infty)$ such that

$$
\mathbb{E}(\exp (-s X(R))) \leq \exp \left(-c R^{(2 v+4)(2 v+3)} s^{1 /(2 v+5)}\right)
$$

for any $s \in[1, \infty)$ and $R \in(0, \bar{R}]$.

In the rest of this section, we prove this lemma. We also represent the Gaussian random magnetic field by

$$
B^{\omega}(x)=B+\int_{\mathbb{R}^{2}} \sigma(x+y) \omega(d y)
$$

as in the previous paper, ${ }^{19}$ where $\omega$ represents the white noise. The condition $v>3 / 2$ is extendible to $v>1$ in the following proof. For any $0<R_{1}<R$, we have

$$
\begin{aligned}
& X(R)-X\left(R_{1}\right)=\int_{R_{1}}^{R} \frac{d r}{2 \pi r} \mid \int_{B\left(R_{1}\right)} d x B^{\omega}(x)+\int_{B(r) \backslash B\left(R_{1}\right)} d x(B \\
& \left.+\int_{(\operatorname{supp} \sigma)^{R_{1}}} \sigma(x+y) \omega(d y)+\int_{\left((\operatorname{supp} \sigma)^{\left.R_{1}\right)^{c}}\right.} \sigma(x+y) \omega(d y)\right)\left.\right|^{2},
\end{aligned}
$$

where $B\left(R_{1}\right)=\left\{x \in \mathbb{R}^{2}:|x|<R_{1}\right)$ and $(\operatorname{supp} \sigma)^{R_{1}}=\left\{x \in \mathbb{R}^{2}: d(x, \operatorname{supp} \sigma)<R_{1}\right\}$. The key point is that $\omega$ on $\left((\operatorname{supp} \sigma)^{R_{1}}\right)^{c}$ is independent of $X\left(R_{1}\right), B^{\omega}(x)$ on $B\left(R_{1}\right)$, and $\omega$ on $(\operatorname{supp} \sigma)^{R_{1}}$. Thus, as in Section 4 of the previous paper, ${ }^{19}$ we have

$$
\mathbb{E}(\exp (-s X(R))) \leq \mathbb{E}\left(\exp \left(-s X\left(R_{1}\right)\right)\right) F\left(R, R_{1} ; s\right),
$$


where

$$
F\left(R, R_{1} ; s\right):=\mathbb{E}\left[\exp \left\{-s X\left(R, R_{1}\right)\right\}\right]
$$

and

$$
X\left(R, R_{1}\right):=\int_{R_{1}}^{R} \frac{d r}{2 \pi r}\left(\int_{B(r) \backslash B\left(R_{1}\right)} d x \int_{\left((\operatorname{supp} \sigma)^{\left.R_{1}\right)^{c}}\right.} \sigma(x+y) \omega(d y)\right)^{2} .
$$

Using the property that the distribution of a centered Gaussian random variable is determined by the variance, as in Section 4 of the previous paper, ${ }^{19}$ we have

$$
F\left(R, R_{1} ; s\right) \leq 1-s \mathbb{E}\left[X\left(R, R_{1}\right)\right]+\frac{3 s^{2}}{2} \mathbb{E}\left[X\left(R, R_{1}\right)\right]^{2} .
$$

Thus, we have only to dominate the integral

$$
\mathbb{E}\left[X\left(R, R_{1}\right)\right]=\int_{R_{1}}^{R} \frac{d r}{2 \pi r} \int_{\left((\operatorname{supp} \sigma)^{\left.R_{1}\right)^{c}}\right.} d y\left(\int_{B(r) \backslash B\left(R_{1}\right)} d x \sigma(x+y)\right)^{2}
$$

from above and below. For the upper estimate, we first use condition (A2) (iii) to obtain

$$
\begin{aligned}
& \mathbb{E}\left[X\left(R, R_{1}\right)\right] \\
\leq & c_{1}^{2} \int_{R_{1}}^{R} \frac{d r}{2 \pi r} \int_{(\operatorname{supp} \sigma)^{r} \backslash(\operatorname{supp} \sigma)^{R_{1}}} d y\left(\int_{(B(y: r) \backslash B(y: d(y, \operatorname{supp} \sigma)) \cap \operatorname{supp} \sigma} d x d(x, \partial \operatorname{supp} \sigma)^{v}\right)^{2},
\end{aligned}
$$

where $B(y: r)=y+B(r)$. By condition (A2) (iv), the previous factor is estimated as

$$
\begin{aligned}
& \int_{(B(y: r) \backslash B(y: d(y, \operatorname{supp} \sigma)) \cap \operatorname{supp} \sigma} d x d(x, \partial \operatorname{supp} \sigma)^{v} \\
= & \int_{d(y, \operatorname{supp} \sigma)}^{r} d s \int_{S^{1}(y: s) \cap \operatorname{supp} \sigma} \mathcal{H}^{1}(d x) d(x, \partial \operatorname{supp} \sigma)^{v} \\
\leq & \int_{d(y, \operatorname{supp} \sigma)}^{r} d s(s-d(y, \operatorname{supp} \sigma))^{v} \mathcal{H}^{1}\left(S^{1}(y: s) \cap \operatorname{supp} \sigma\right) \\
\leq & c_{2} \int_{d(y, \operatorname{supp} \sigma)}^{r} d s(s-d(y, \operatorname{supp} \sigma))^{v+1 / 2},
\end{aligned}
$$

where $S^{1}(y: s)=\left\{x \in \mathbb{R}^{2}:|x-y|=s\right\}$. Thus, we use also (A2) (ii) to obtain

$$
\begin{aligned}
& \mathbb{E}\left[X\left(R, R_{1}\right)\right] \leq c_{6} \int_{R_{1}}^{R} \frac{d r}{r} \int_{(\operatorname{supp} \sigma)^{r} \backslash(\operatorname{supp} \sigma)^{R_{1}}} d y\left(\int_{0}^{r-d(y, \operatorname{supp} \sigma)} s^{v+1 / 2} d s\right)^{2} \\
& =c_{7} \int_{R_{1}}^{R} \frac{d r}{r} \int_{(\operatorname{supp} \sigma)^{r} \backslash(\operatorname{supp} \sigma)^{R_{1}}} d y(r-d(y, \operatorname{supp} \sigma))^{2 v+3} \\
& =c_{7} \int_{R_{1}}^{R} \frac{d r}{r} \int_{R_{1}}^{r} d t(r-t)^{2 v+3} \int_{\left\{y \in \mathbb{R}^{2}: d(y, \operatorname{supp} \sigma)=t\right\}} \frac{\mathcal{H}^{1}(d y)}{|\nabla d(y, \operatorname{supp} \sigma)|} \\
& \leq c_{8} \int_{R_{1}}^{R} \frac{d r}{r} \int_{R_{1}}^{r} d t(r-t)^{2 v+3}=\frac{c_{9}}{\left\langle R, R_{1}\right\rangle_{2 v+4}}\left(R-R_{1}\right)^{2 v+5},
\end{aligned}
$$

where

$$
\left\langle R, R_{1}\right\rangle_{2 v+4}:=\left(\int_{0}^{1} \frac{(2 v+5) r^{2 v+4} d r}{R_{1}+r\left(R-R_{1}\right)}\right)^{-1}
$$

is a harmonic mean of the probability distribution $(2 v+5) r^{2 v+4} d r$ on the interval $\left[R_{1}, R\right]$. For the lower estimate, we first use condition (A2) (i) to restrict as

$$
\mathbb{E}\left[X\left(R, R_{1}\right)\right] \geq \int_{R_{1}}^{R} \frac{d r}{2 \pi r} \int_{\Sigma\left(\theta_{*}, \varepsilon_{1}, \varepsilon_{2}\right) \backslash(\operatorname{supp} \sigma)^{R_{1}}} d y\left|\int_{B(y, r) \backslash B(y, d(y, \operatorname{supp} \sigma))} d x \sigma(x)\right|^{2} .
$$


By assuming $R \leq \varepsilon_{1}$ and using condition (A2) (v), the last factor is estimated as

$$
\begin{gathered}
\int_{B(y, r) \backslash B(y, d(y, \operatorname{supp} \sigma))} d x \sigma(x)=\int_{d(y, \operatorname{supp} \sigma)}^{r} d t \int_{S^{1}(y: t)} \mathcal{H}^{1}(d x) \sigma(x) \\
\geq c_{10} \int_{d(y, \operatorname{supp} \sigma)}^{r} d t(t-d(y, \operatorname{supp} \sigma))^{v+1 / 2} \geq c_{11}(r-d(y, \operatorname{supp} \sigma))^{v+3 / 2} .
\end{gathered}
$$

Next, we use also (ii) and (vi) in condition (A2) to obtain

$$
\begin{aligned}
\mathbb{E}\left[X\left(R, R_{1}\right)\right] & \geq c_{12} \int_{R_{1}}^{R} \frac{d r}{r} \int_{\Sigma\left(\theta_{*}, \varepsilon_{1}, \varepsilon_{2}\right)(\operatorname{supp} \sigma)^{R_{1}}} d y(r-d(y, \operatorname{supp} \sigma))_{+}^{2 v+3} \\
& =c_{12} \int_{R_{1}}^{R} \frac{d r}{r} \int_{R_{1}}^{r} d t(r-t)^{2 v+3} \int_{\left\{y \in \Sigma\left(\theta_{*}, \varepsilon_{1}, \varepsilon_{2}\right): d(y, \operatorname{supp} \sigma)=t\right\}} \frac{\mathcal{H}^{1}(d y)}{|\nabla d(y, \operatorname{supp} \sigma)|} \\
& \geq c_{13} \int_{R_{1}}^{R} \frac{d r}{r} \int_{R_{1}}^{r} d t(r-t)^{2 v+3}=\frac{c_{14}}{\left\langle R, R_{1}\right\rangle_{2 v+4}}\left(R-R_{1}\right)^{2 v+5} .
\end{aligned}
$$

Eqs. (46) and (50) mean that their right-hand sides are the leading terms up to constants.

As in the proof of Lemma 4.2 in the previous paper, ${ }^{19}$ we take $\left\{R_{j}\right\}_{j}$ as follows: taking $\varepsilon \in(0,1 / 2)$ and introducing the sequence

$$
b_{k}:=\left\{\begin{array}{l}
\frac{1-2 \varepsilon}{(1-\varepsilon)\lceil 1 / \varepsilon\rceil} \text { for } k \in[0,\lceil 1 / \varepsilon\rceil] \cap \mathbb{N}, \\
\varepsilon^{k-\lceil 1 / \varepsilon\rceil} \text { for } k \in(\lceil 1 / \varepsilon\rceil, \infty) \cap \mathbb{N},
\end{array}\right.
$$

whose elements are in $(0, \varepsilon)$ and whose sum is 1 , we set $R_{0}=R$ and

$$
R_{j-1}-R_{j}=\frac{b_{k} R}{\left\lfloor R s^{1 /(2 v+5)}\right\rfloor} \text { for } j \in\left((k-1)\left\lfloor R s^{1 /(2 v+5)}\right\rfloor, k\left\lfloor R s^{1 /(2 v+5)}\right\rfloor\right] \cap \mathbb{N},
$$

where $\lfloor a\rfloor=\max \{(-\infty, a] \cap \mathbb{Z}\}$ and $\lceil a\rceil=\min \{[a, \infty) \cap \mathbb{Z}\}$ for any $a \in \mathbb{R}$. For

$$
F\left(R_{j-1}, R_{j}: s\right) \leq 1-\frac{s c_{14}}{2\left\langle R_{j-1}, R_{j}\right\rangle_{2 v+4}}\left(R_{j-1}-R_{j}\right)^{2 v+5}
$$

and

$$
\log F\left(R_{j-1}, R_{j}: s\right) \leq-\frac{s c_{14}}{2\left\langle R_{j-1}, R_{j}\right\rangle_{2 v+4}}\left(R_{j-1}-R_{j}\right)^{2 v+5}
$$

to be satisfied, it suffices that

$$
s\left(R_{j-1}-R_{j}\right)^{2 v+5} \leq c_{15} R_{j}
$$

for some $c_{15} \in(0, \infty)$, and subsequently

$$
\varepsilon \leq c_{16} R^{1 /(2 v+3)}
$$

for some $c_{16} \in(0, \infty)$. Then we have

$$
\begin{aligned}
& \log \mathbb{E}[\exp (-s X(R))] \leq \sum_{j=1}^{\infty} \log F\left(R_{j-1}, R_{j}: s\right) \\
\leq & -s c_{17} \sum_{j=1}^{\infty} \frac{\left(R_{j-1}-R_{j}\right)^{2 v+5}}{R_{j-1}} \leq-c_{18} \varepsilon^{2 v+4} s^{1 /(2 v+5)} .
\end{aligned}
$$

For the above first inequality, we need only $X(R) \geq \sum_{j}\left(X\left(R_{j-1}\right)-X\left(R_{j}\right)\right)$. By taking $\varepsilon$ as $c_{16} R^{1 /(2 v+3)}$, we can complete the proof. 


\section{PROOF OF THEOREM 1}

We first note that our Cameron-Martin space $L^{2}\left(\mathbb{R}^{2}\right) \oplus L^{2}\left(\mathbb{R}^{2}\right)$ may be replaced by a smaller space,

$$
\left(\begin{array}{c}
\operatorname{Re} \\
\operatorname{Im}
\end{array}\right) \mathcal{F} L^{2}\left(\Lambda_{L+\bar{\Sigma}}\right)
$$

by Eq. (9), where $\bar{\Sigma}=2 \sup _{x, y \in \operatorname{supp} \sigma}|x-y|_{\infty}$ and $\mathcal{F}$ is the Fourier transform on $L^{2}\left(\mathbb{R}^{2}\right)$. As its complete orthonormal basis, we take

$$
\boldsymbol{\Phi}_{\boldsymbol{n}}:=\left(\begin{array}{c}
\operatorname{Re} \\
\operatorname{Im}
\end{array}\right) \widehat{\Phi_{n, L+\bar{\Sigma}}}
$$

for $\boldsymbol{n} \in \mathbb{N}^{2}$, where $\Phi_{\boldsymbol{n}, L+\bar{\Sigma}}$ is the function appeared in Eq. (14), where $L$ is replaced by $L+\bar{\Sigma}$. Then, we have

$$
\left\|D \lambda_{\ell}\left(H_{L}^{\omega}\right)\right\|_{L^{2}\left(\mathbb{R}^{2}\right) \oplus L^{2}\left(\mathbb{R}^{2}\right)}^{2}=\sum_{\boldsymbol{n} \in \mathbb{N}^{2}}\left(D_{\boldsymbol{\Phi}_{\boldsymbol{n}}} \lambda_{\ell}\left(H_{L}^{\omega}\right)\right)^{2},
$$

where $D_{\boldsymbol{\Phi}_{\boldsymbol{n}}} \lambda_{\ell}\left(H_{L}^{\omega}\right):=\left(D \lambda_{\ell}\left(H_{L}^{\omega}\right), \boldsymbol{\Phi}_{\boldsymbol{n}}\right)_{L^{2}\left(\mathbb{R}^{2}\right) \oplus L^{2}\left(\mathbb{R}^{2}\right)}$. We modify Lemma 1.1 so that the left-hand side of Eq. (10) is replaced by a partial finite sum of the right-hand side of the preceding equation. In the previous paper, ${ }^{19}$ this step was done in Section II. Because

$$
\begin{aligned}
& D_{\boldsymbol{\Phi}_{\boldsymbol{n}}} \lambda_{\ell}\left(H_{L}^{\omega}\right) \\
= & \operatorname{Re} \int_{\mathbb{R}^{2}} d \xi \overline{\widehat{\sigma}(\xi)} \widehat{\Phi_{\boldsymbol{n}, L+\bar{\Sigma}}}(\xi) \int_{\Lambda_{L}} d y \exp (i 2 \pi y \cdot \xi) \sum_{\boldsymbol{n}^{\prime} \in \mathbb{N}^{2}} \frac{\Phi_{\boldsymbol{n}^{\prime}, L}(y)}{E_{\boldsymbol{n}^{\prime}, L}}\left(\Phi_{\boldsymbol{n}^{\prime}, L}, \nabla \times j_{L}^{\omega}\right)_{L^{2}\left(\Lambda_{L}\right)},
\end{aligned}
$$

we have

$$
\begin{aligned}
& \sum_{\boldsymbol{n} \in \mathbb{N}^{2}:|\boldsymbol{n}|>\mathcal{R}}\left(D_{\boldsymbol{\Phi}_{\boldsymbol{n}}} \lambda_{\ell}\left(H_{L}^{\omega}\right)\right)^{2} \\
\leq & \frac{1}{\mathcal{R}^{4}} \sum_{\boldsymbol{n} \in \mathbb{N}^{2}}|\boldsymbol{n}|^{4}\left|\int_{\mathbb{R}^{2}} d \xi \overline{\widehat{\sigma}(\xi)} \widehat{\Phi_{\boldsymbol{n}, L+\bar{\Sigma}}}(\xi) \int_{\Lambda_{L}} d y \exp (i 2 \pi y \cdot \xi) \sum_{\boldsymbol{n}^{\prime} \in \mathbb{N}^{2}} \frac{\Phi_{\boldsymbol{n}^{\prime}, L}(y)}{E_{\boldsymbol{n}^{\prime}, L}}\left(\Phi_{\boldsymbol{n}^{\prime}, L}, \nabla \times j_{L}^{\omega}\right)_{L^{2}\left(\Lambda_{L}\right)}\right|^{2}
\end{aligned}
$$

for any $\mathcal{R} \in(0, \infty)$. Moreover, as

$$
|\boldsymbol{n}|^{2} \Phi_{\boldsymbol{n}, L+\bar{\Sigma}}(\xi)=\left(\frac{L+\bar{\Sigma}}{\pi}\right)^{2}|2 \pi \xi|^{2} \Phi_{\boldsymbol{n}, L+\bar{\Sigma}}(\xi) \text { and } \frac{-\Delta \Phi_{\boldsymbol{n}^{\prime}, L}}{E_{\boldsymbol{n}^{\prime}, L}}=\Phi_{\boldsymbol{n}^{\prime}, L},
$$

the right-hand side is

$$
\begin{aligned}
& \left(\frac{L+\bar{\Sigma}}{\pi \mathcal{R}}\right)^{4} \sum_{\boldsymbol{n} \in \mathbb{N}^{2}}\left|\int_{\mathbb{R}^{2}} d \xi \widehat{\Phi_{\boldsymbol{n}, L+\bar{\Sigma}}}(\xi) \overline{\widehat{\sigma}(\xi)\left(\widehat{\nabla \times j_{L}^{\omega}}\right)(\xi)}\right|^{2} \\
\leq & \left(\frac{L+\bar{\Sigma}}{\pi \mathcal{R}}\right)^{4} \int_{\mathbb{R}^{2}} d \xi\left|\widehat{\sigma}(\xi)\left(\widehat{\nabla \times j_{L}^{\omega}}\right)(\xi)\right|^{2} \\
\leq & \left(\frac{L+\bar{\Sigma}}{\pi \mathcal{R}}\right)^{4}\|\sigma\|_{L^{1}\left(\mathbb{R}^{2}\right)}^{2}\left\|\nabla \times j_{L}^{\omega}\right\|_{L^{2}\left(\Lambda_{L}\right)}^{2} .
\end{aligned}
$$

The quantity $\left\|\nabla \times j_{L}^{\omega}\right\|_{L^{2}\left(\Lambda_{L}\right)}^{2}$ was dominated in Lemma 3.1 of the previous paper. ${ }^{19}$ Hence, we obtain

$$
\begin{aligned}
& \sum_{\boldsymbol{n} \in \mathbb{N}^{2}:|\boldsymbol{n}| \leq \mathcal{R}}\left(D_{\boldsymbol{\Phi}_{\boldsymbol{n}}} \lambda_{\ell}\left(H_{L}^{\omega}\right)\right)^{2} \geq \frac{c_{1}}{L^{m_{1}}\left(\left\|B^{\omega}\right\|_{W^{2,2}\left(\Lambda_{L}\right)}^{2}+R\right)^{m_{2}}} X_{\omega}\left(\frac{c_{2}}{\left.L^{m_{3}\left(\left\|B^{\omega}\right\|_{W^{2,2}\left(\Lambda_{L}\right)}^{2}+R\right)^{m_{4}}}, x_{\omega}\right)^{m_{5}}}\right. \\
& -c_{3} L^{22}\left(\left\|B^{\omega}\right\|_{W^{2,2}\left(\Lambda_{L}\right)}^{2}+R\right)^{4} / \mathcal{R}^{4} .
\end{aligned}
$$

In this inequality and in the following, $\left\{c_{j}\right\}_{j=1,2, \ldots}$ are the universal constants distinct from those in Secs. I-III. Therefore, we obtain 


$$
\begin{aligned}
& \sum_{\boldsymbol{n} \in \mathbb{N}^{2}:|\boldsymbol{n}| \leq \mathcal{R}(\omega)}\left(D_{\boldsymbol{\Phi}_{\boldsymbol{n}}} \lambda_{\ell}\left(H_{L}^{\omega}\right)\right)^{2}
\end{aligned}
$$

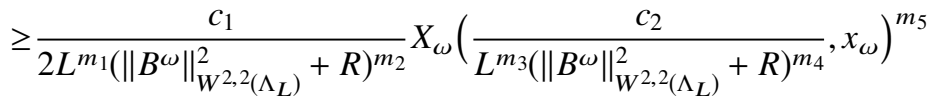

by taking $\mathcal{R}$ as

$$
\mathcal{R}(\omega)=\left\{\frac{2 c_{3} L^{m_{1}+22}\left(\left\|B^{\omega}\right\|_{W^{2,2}\left(\Lambda_{L}\right)}^{2}+R\right)^{m_{2}+4}}{c_{1} X_{\omega}\left(\frac{c_{2}}{\left.L^{m_{3}\left(\left\|B^{\omega}\right\|_{W^{2,2}\left(\Lambda_{L}\right)}^{2}\right.}\right)^{m_{4}}}, x_{\omega}\right)^{m_{5}}}\right\}^{1 / 4} .
$$

Thus, we modify the first estimate in Section 5 of the previous paper ${ }^{19}$ as

$$
\begin{aligned}
& \operatorname{Tr}\left[\chi_{[E-\eta, E+\eta]}\left(H_{L}^{\omega}\right)\right] \\
& \leq c_{4} \sum_{\ell} \chi_{[(t(E)-\eta) \vee t(0), t(E)+\eta]}\left(t\left(\lambda_{\ell}\left(H_{L}^{\omega}\right)\right)\right) L^{m_{1}} \\
& \times \sum_{m \in \mathbb{N}} \widetilde{\chi}_{[m-1, m]}\left(\left\|B^{\omega}\right\|_{W^{2,2}\left(\Lambda_{L}\right)}^{2}+R\right) m^{m_{2}}
\end{aligned}
$$

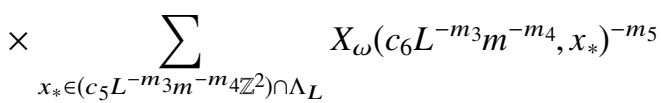

$$
\begin{aligned}
& \times \sum_{\boldsymbol{n} \in \mathbb{N}^{2}} \tilde{\chi}_{[0, \infty)}\left(\mathcal{R}\left(m, x_{*}, \omega\right)-|\boldsymbol{n}|\right)\left(D_{\boldsymbol{\Phi}_{\boldsymbol{n}}} t\left(\lambda_{\ell}\left(H_{L}^{\omega}\right)\right)\right)^{2},
\end{aligned}
$$

where $t(u):=(u+1)(5 R)^{3} /(5 R+u+1)^{3}$,

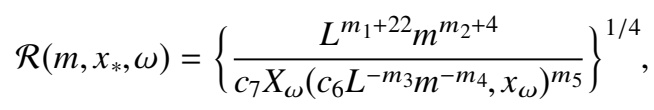

and, for each interval $I, \widetilde{\chi}_{I}$ is a [0,1]-valued smooth function on $\mathbb{R}$ such that $\tilde{\chi}_{I}=1$ on $I$ and $\widetilde{\chi}_{I}(x)=0$ if $\operatorname{dist}(x, I) \geq 1$. As in the previous paper, ${ }^{19}$ we next use

$$
\begin{aligned}
& \sum_{\ell} \chi_{[(t(E)-\eta) \vee t(0), t(E)+\eta]}\left(t\left(\lambda_{\ell}\left(H_{L}^{\omega}\right)\right)\right)\left(D_{\boldsymbol{\Phi}_{\boldsymbol{n}}} t\left(\lambda_{\ell}\left(H_{L}^{\omega}\right)\right)\right)^{2} \\
\leq & D_{\boldsymbol{\Phi}_{\boldsymbol{n}}}^{2} \operatorname{Tr}\left[G\left(t\left(H_{L}^{\omega}\right)\right)\right]-\operatorname{Tr}\left[F\left(t\left(H_{L}^{\omega}\right)\right) D_{\boldsymbol{\Phi}_{\boldsymbol{n}}}^{2} t\left(H_{L}^{\omega}\right)\right]
\end{aligned}
$$

and estimate each term on the right-hand side under the condition

$$
\left\|B^{\omega}\right\|_{W^{2,2}\left(\Lambda_{L}\right)}^{2}+R \in[m-2, m+1],
$$

where $F$ and $G$ are the functions on $\mathbb{R}$ such that $F^{\prime}=\chi_{[(t(E)-\eta) \vee t(0), t(E)+\eta]}, G^{\prime}=F$, and $F=G=0$ on $(-\infty,(t(E)-\eta) \vee t(0)]$. For this, we first prove

$$
\left\|D_{\mathbf{\Phi}_{\boldsymbol{n}}}^{2} t\left(H_{L}^{\omega}\right)\right\|_{L^{2}\left(\Lambda_{L}\right) \rightarrow L^{2}\left(\Lambda_{L}\right)} \leq c_{8} L^{6}
$$

using

$$
\left\|D_{\boldsymbol{\Phi}_{\boldsymbol{n}}} A_{L}^{\omega}\right\|_{L^{\infty}\left(\Lambda_{L}\right)} \leq c_{9} L^{3} .
$$

Eq. (73) is proven by Lemma 3.3 in the previous paper ${ }^{19}$ and condition (A1) (i) as follows:

$$
\begin{aligned}
& \left\|D_{\boldsymbol{\Phi}_{\boldsymbol{n}}} A_{L}^{\omega}\right\|_{L^{\infty}\left(\Lambda_{L}\right)} \leq c_{10} L\left\|D_{\boldsymbol{\Phi}_{\boldsymbol{n}}} B^{\omega}\right\|_{W^{2,1}\left(\Lambda_{L}\right)} \\
\leq & c_{11} L \sum_{\boldsymbol{\alpha} \in \mathbb{Z}_{+}^{2}:|\alpha|_{1} \leq 2} \int_{\Lambda_{L}} d x\left|\int \exp (i 2 \pi x \cdot \xi)(2 \pi \xi)^{\alpha} \overline{\widehat{\sigma}(\xi)} \widehat{\boldsymbol{\Phi}_{\boldsymbol{n}, L+\bar{\Sigma}}}(\xi) d \xi\right| \\
\leq & c_{11} L \sum_{\boldsymbol{\alpha} \in \mathbb{Z}_{+}^{2}:|\alpha|_{1} \leq 2}\left|\Lambda_{L}\right|\left(\int\left|(2 \pi \xi)^{\alpha}\right|^{2} \widehat{\beta}(\xi) d \xi\right)^{1 / 2}\left\|\boldsymbol{\Phi}_{\boldsymbol{n}, L+\bar{\Sigma}}\right\| .
\end{aligned}
$$

The rest of the proof of Eq. (72) is simpler than the corresponding part in the previous paper ${ }^{19}$ because $D_{\boldsymbol{\Phi}_{\boldsymbol{n}}}^{2} A_{L}^{\omega}=0$. 
Moreover, the remaining proof is also simpler than the corresponding part in the previous paper ${ }^{19}$ because our direction $\boldsymbol{\Phi}_{\boldsymbol{n}}$ of the $H$-differentiation is independent of $\omega$; this independence simplifies the application of the integration by parts on the Wiener space used to remove the $H$-differentiation from $\operatorname{Tr}\left[G\left(t\left(H_{L}^{\omega}\right)\right)\right]$.

\section{LIFSHITZ TAIL}

As stated in Section I, the corollary of Theorem 1 is proven using the results regarding the Lifshitz tail owing to Nakamura ${ }^{12}$ and the theory of multi-scale analysis by Germinet and Klein. ${ }^{8}$ However, the Lifshitz-tail results in Nakamura ${ }^{12}$ seem not to be applicable to the setting of this paper because boundedness of the magnetic field is assumed in Nakamura. ${ }^{12}$

In this section, we demonstrate that boundedness is not necessary and the results in Nakamura ${ }^{12}$ are extendible to a general setting including the Gaussian random magnetic field. Furthermore, we treat the arbitrary dimensional setting: on a $d$-dimensional Euclidean space $\mathbb{R}^{d}$ and a general probability space $(\Omega, \mathcal{F}, \mathbb{P})$, we consider a $d \times d$ skew-symmetric matrix-valued random field $B^{\omega}=\left(B_{j k}^{\omega}(x)\right)_{1 \leq j, k \leq d, x \in \mathbb{R}^{d}, \omega \in \Omega}$ satisfying the following:

(A3) (i) There exists on the probability space a group of metrically transitive transformations $\left\{T_{x}\right\}_{x \in \mathbb{R}^{d}}$ on $\Omega$ such that $B^{T_{x} \omega}(y)=B^{\omega}(x+y)$ for any $x, y \in \mathbb{R}^{d}$.

(ii) For each $\omega \in \Omega$, the map $\mathbb{R}^{d} \ni x \mapsto B^{\omega}(x)$ is locally $p$ th integrable, where $p=2$.

(iii) For each $\omega \in \Omega$, the 2-form $\mathcal{B}^{\omega}=\sum_{j, k} B_{j k}^{\omega}(x) d x_{j} \wedge d x_{k}$ is closed in the sense of distributions.

(iv) There exists a nonnegative decreasing function $\varphi$ on the interval $[0, \infty)$ satisfying $\varphi(t) \rightarrow 0$ as $t \rightarrow \infty$ and the following: for any bounded subsets $\Lambda_{1}, \Lambda_{2}$ of $\mathbb{R}^{d}, \Sigma_{\Lambda_{1}}$-measurable essentially bounded function $g$ and $\Sigma_{\Lambda_{2}}$-measurable integrable function $f$ on $\Omega$, we have

$$
|\mathbb{E}(f g)-\mathbb{E}(f) \mathbb{E}(g)| \leq \varphi\left(d\left(\Lambda_{1}, \Lambda_{2}\right)\right)\|f\|_{L^{1}}\|g\|_{L^{\infty}},
$$

where $d(\cdot, \cdot)$ is the Euclidean distance and $\Sigma_{\Lambda}$ is the $\sigma$-algebra generated by $\left\{B^{\omega}(x) \mid x \in \Lambda\right\}$ for any $\Lambda \subset \mathbb{R}^{d}$.

(v) There exist $r \in(0, \infty)$ and $1 \leq j<k \leq d$ such that

$$
\mathbb{P}\left(\frac{1}{2 \pi} \int_{D_{j k}(r)} B_{j k}^{\omega}(y) \mathcal{H}^{2}(d y) \notin \mathbb{Z}\right)>0,
$$

where $\mathcal{H}^{2}$ is the two-dimensional Hausdorff measure and $D_{j k}(r)=\left\{y \in \mathbb{R}^{d}: y_{j}^{2}+\right.$ $y_{k}^{2} \leq r^{2}, y_{h}=0$ for $\left.h \neq j, k\right\}$.

Under assumptions (A3) (ii) and (iii), there exists an $\mathbb{R}^{d}$-valued random field $A_{L}^{\omega}$ $=\left(A_{L, j}^{\omega}(x)\right)_{1 \leq j \leq d, x \in \Lambda_{L}, \omega \in \Omega}$ for each $L>0$ such that $\Lambda_{L} \ni x \mapsto A_{L}^{\omega}(x)$ is square integrable and is a vector potential of the magnetic field $\mathcal{B}^{\omega}: d \sum_{j=1}^{d} A_{L, j}^{\omega}(x) d x_{j}=\mathcal{B}^{\omega}$ on $\Lambda_{L}$, where $d$ on the left-hand side is the exterior derivative in the sense of distributions and $\Lambda_{L}=(-L / 2, L / 2)^{d}$. Indeed,

$$
A_{L, j}^{\omega}(x)=\int_{0}^{1} \sum_{k=1}^{d}\left(\chi_{\Lambda_{L}} B_{k j}^{\omega}\right)\left(x^{L}+t\left(x-x^{L}\right)\right) t\left(x_{k}-x_{k}^{L}\right) d t
$$

is one of the random fields satisfying these, where $x_{L}$ is an arbitrary fixed point apart from $\Lambda_{L}$. We can then define self-adjoint operators

$$
\mathbf{H}_{L}^{\omega, \#}:=\sum_{\iota=1}^{d}\left(i \partial_{\iota}+A_{L, \iota}^{\omega}(x)\right)^{2}
$$

on $L^{2}\left(\Lambda_{L}\right)$ with the Dirichlet and the Neumann boundary conditions for $\#=D$ and $\#=N$, respectively. For these operators, let $N\left(E ; \mathbf{H}_{L}^{\omega, \#}\right)$ be the numbers of eigenvalues not exceeding $E$. These numbers are determined only by the magnetic field $B^{\omega}$ and are independent of the choice of $A_{L}^{\omega}$ by 
gauge invariance. Then, by condition (A3) (i), the well-known limit

$$
\frac{1}{\left|\Lambda_{L}\right|} N\left(E ; \mathbf{H}_{L}^{\omega, \#}\right) \longrightarrow N(E) \quad \text { as } L \rightarrow \infty
$$

exists for almost every $\omega$ and defines a deterministic increasing function $N(E)$ independent of $\# \in\{D, N\}$ (cf. Carmona and Lacroix, ${ }^{1}$ Doi, Iwatsuka, and Mine, ${ }^{2}$ Pastur and Figotin, ${ }^{14}$ Ueki $^{17}$ ). This is the integrated density of states for a Schrödinger operator with the magnetic field $B^{\omega}$. To represent the operator globally, we need a vector potential defined on $\mathbb{R}^{d}$. For this it suffices to assume $p>d / 2$ in condition (A3) (ii). Indeed, under this assumption, the $\mathbb{R}^{d}$-valued random field

$$
A_{j}^{\omega}(x)=\int_{0}^{1} \sum_{k=1}^{d} B_{k j}^{\omega}(t x) t x_{k} d t
$$

gives the vector potential such that the map $\mathbb{R}^{d} \ni x \mapsto A^{\omega}(x)$ is locally square integrable. Then, by Leinfelder and Simader, ${ }^{11}$ the operator

$$
\mathbf{H}^{\omega}:=\sum_{\iota=1}^{d}\left(i \partial_{\iota}+A_{\iota}^{\omega}(x)\right)^{2}
$$

is essentially self-adjoint on $C_{0}^{\infty}\left(\mathbb{R}^{d}\right)$, and $(N(E))_{E \in[0, \infty)}$ is its integrated density of states.

The main statement in this section is then the following:

Theorem 2 (Lifshitz tail). Under condition (A3), the integrated density of states $(N(E))_{E \in[0, \infty)}$ defined by Eq. (77) satisfies

$$
\varlimsup_{E \downarrow 0} \frac{\log (-\log N(E))}{\log E} \leq-\frac{d}{2} .
$$

To prove this, we use the following:

Proposition 5.1 (cf. Theorem 2 in Nakamura ${ }^{12}$ ). For any $r \in(0, \infty)$, we define a function on $\mathbb{R}^{d}$ by

$$
W^{\omega}(x ; r)=\frac{2}{d(d-1) \pi r^{3}} \sum_{1 \leq j<k \leq d} \int_{\partial D_{j k}(r)} \mathcal{H}^{1}(d z) d\left(\frac{1}{2 \pi} \int_{D_{j k}(r)} \mathcal{H}^{2}(d y) B_{j k}^{\omega}(x+y+z), \mathbb{Z}\right)^{2},
$$

where $d(a, \mathbb{Z})$ is the distance to the set of integers from a for any $a \in \mathbb{R}$. Then, we have

$$
\left(\psi, \mathbf{H}_{L}^{\omega, N} \psi\right) \geq\left(\psi, W^{\omega}(\cdot ; r) \psi\right)
$$

for any $\psi \in \operatorname{Dom}\left(\mathbf{H}_{L}^{\omega, N}\right)$ and $L, r>0$.

The proof of this proposition is the same as that of Theorem 2 in Nakamura. ${ }^{12}$

Proof of Theorem 2. Clearly $W^{\omega}(x ; r)$ is bounded; it is also positive with a positive probability under condition (A3) (v). Thus, by condition (A3) (iv), Theorem 4 in Kirsch and Martinelli ${ }^{10}$ applies to the Schrödinger operator $\left(-\Delta+W^{\omega}(\cdot ; r)\right) / 2$ without change, and its integrated density of states $N_{1}(E)$ satisfies $\varlimsup_{E \downarrow 0} E^{d / 2} \log N_{1}(E)<0$. By the Abelian theorem, its Laplace-Stieltjes transform $\widetilde{N}_{1}(t)=\int_{0}^{\infty} e^{-t E} d N_{1}(E)$ satisfies $\varlimsup_{t \rightarrow \infty} t^{-d /(d+2)} \log \widetilde{N}_{1}(t)<0$ (cf. Lemma 3.2 (ii) in Ueki ${ }^{18}$ ). Let $N_{2}(E)$ be the integrated density of states obtained by

$$
\frac{1}{\left|\Lambda_{L}\right|} N\left(E ;\left(\mathbf{H}_{L}^{\omega, \#}+W^{\omega}(\cdot ; r)\right) / 2\right) \longrightarrow N_{2}(E) \quad \text { as } L \rightarrow \infty .
$$

Then, by the Feynman-Kac-Itô formula and the stochastic Stokes theorem, its Laplace-Stieltjes transform $\widetilde{N_{2}}(t)$ is represented as

$$
\begin{aligned}
\widetilde{N}_{2}(t)=\mathbb{E}[ & \exp \left(-i \sum_{1 \leq j<k \leq d} \int_{0}^{t} d S_{j k}(s) \int_{0}^{1} d v 2 v B_{j k}^{\omega}(v w(s))\right. \\
& \left.\left.-\frac{1}{2} \int_{0}^{t} W^{\omega}(w(s) ; r) d s\right) \mid w(t)=0\right] \frac{1}{(2 \pi t)^{d / 2}}
\end{aligned}
$$


where $w(s)=\left(w_{1}(s), w_{2}(s), \ldots, w_{d}(s)\right)$ is the $d$-dimensional Wiener process starting at the origin and

$$
S_{j k}(t)=\frac{1}{2} \int_{0}^{t}\left(w_{j}(s) d w_{k}(s)-w_{k}(s) d w_{j}(s)\right)
$$

By taking the absolute value of the integrand, we have $\widetilde{N}_{2}(t) \leq \widetilde{N}_{1}(t)$. Hence we have $\overline{\lim }_{t \rightarrow \infty} t^{-d /(d+2)}$ $\log \widetilde{N}_{2}(t)<0$. By the Tauberian theorem, we have $\varlimsup_{\lim }^{E \downarrow 0} E^{d / 2} \log N_{2}(E)<0$ (cf. Lemma 3.2 (i) in Ueki $\left.{ }^{18}\right)$. By Proposition 5.1, we have $N_{2}(E) \leq N(E)$. Therefore, we obtain $\overline{\lim }_{E \downarrow 0} E^{d / 2} \log N(E)<0$ and we can complete the proof.

In Nakamura, ${ }^{12}$ the boundedness of the magnetic field was assumed to reduce the operator $\left(-\Delta+W^{\omega}(\cdot ; r)\right) / 2$ without using the Laplace-Stieltjes transform of the integrated density of states.

\section{ACKNOWLEDGMENTS}

The author is partially supported by the Grant-in-Aid for Scientific Research (C) \#26400132 from JSPS.

\section{APPENDIX A: UNIFORM NONDEGENERACY OF THE ZEROS OF THE BESSEL FUNCTIONS}

The Fourier transform of the functions $\sigma$ in Example 1.1 is expressed in terms of the Bessel functions. Indeed, in (i), by taking the appropriate coordinate, the function is expressed as

$$
\sigma(x)=\left(\bar{\sigma}^{2}-\sum_{j=1}^{2}\left(a_{j} x_{j}\right)^{2}\right)_{+}^{v},
$$

where $\left\{a_{j}^{2}\right\}_{j=1,2}$ are the eigenvalues of the matrix $\left(a_{i j}\right)_{1 \leq i, j \leq 2}$. Therefore, as in Section 2 of the previous paper, ${ }^{19}$ its Fourier transform is expressed as

$$
\widehat{\sigma}(\xi)=\frac{\bar{\sigma}^{v+1} \Gamma(v+1)}{\left|a_{1} a_{2}\right| \pi^{v}\left|\left(\xi_{1} / a_{1}, \xi_{2} / a_{2}\right)\right|^{v+1}} J_{v+1}\left(2 \pi \bar{\sigma}\left|\left(\frac{\xi_{1}}{a_{1}}, \frac{\xi_{2}}{a_{2}}\right)\right|\right),
$$

where

$$
J_{v+1}(t)=\sum_{m=0}^{\infty} \frac{(-1)^{m}(t / 2)^{2 m+v+1}}{m ! \Gamma(m+v+2)}
$$

is the Bessel function of order $v+1$ (cf. Abramowitz and Stegun 9 9.1.18 and 11.4.10). In (ii), if $\sigma_{1}(x)=|x|^{\nu_{1}}\left(\overline{\sigma_{1}}-|x|\right)_{+}^{\nu_{1}}$ and $\sigma_{2}(x)=\left({\overline{\sigma_{2}}}^{2}-x^{2}\right)_{+}^{\nu_{2}}$, then their Fourier transforms are

$$
\widehat{\sigma_{1}}(\xi)=\frac{{\overline{\sigma_{1}}}^{v_{1}+1 / 2} \Gamma\left(v_{1}+1\right)}{2^{2 v_{1}} \pi^{v_{1}}|\xi|^{\nu_{1}+1 / 2}} \cos \left(2 \pi \overline{\sigma_{1}}|\xi|\right) J_{v_{1}+1 / 2}\left(2 \pi \overline{\sigma_{1}}|\xi|\right)
$$

and

$$
\widehat{\sigma_{2}}(\xi)=\frac{{\overline{\sigma_{2}}}^{v_{2}+1 / 2} \Gamma\left(v_{2}+1\right)}{\pi^{v_{2}}|\xi|^{\nu_{2}+1 / 2}} J_{v_{2}+1 / 2}\left(2 \pi \overline{\sigma_{2}}|\xi|\right)
$$

(cf. Erdélyi, Magnus, Oberhettinger, and Tricomi ${ }^{3} 1.3 .4$ and 1.3.8).

To prove that these examples satisfy condition (A1) (ii), we use the following:

Proposition A.1. There exist finite positive constants $c_{1}$ and $c_{2}$ depending only on $v$ such that

$$
\left|J_{v}\left(j_{v, s}+z\right)\right|\left|j_{v, s}+z\right|^{c_{1}} \geq c_{2}|z|
$$

for any $s \in \mathbb{N}$ and $z \in\left(\left(j_{v, s-1}-j_{v, s}\right) / 2,\left(j_{v, s+1}-j_{v, s}\right) / 2\right)$, where, for any $s \in \mathbb{N}, j_{n u, s}$ is the sth positive zero of the Bessel function $J_{v}$, and $j_{v, 0}=-j_{v, 1}$. 
Proof. We first use the infinite products representation

$$
J_{v}(z)=\frac{(z / 2)^{v}}{\Gamma(v+1)} \prod_{s=1}^{\infty}\left\{1-\left(\frac{z}{j_{v, s}}\right)^{2}\right\}
$$

and the asymptotic behavior, i.e., for any $\delta>0$, there exists $s_{*} \in \mathbb{N}$ such that

$$
\left|j_{v, s}-\left(s+\frac{v}{2}-\frac{1}{4}\right) \pi\right| \leq \delta / 2
$$

for any $s_{*} \leq s \in \mathbb{N}$ (cf. Abramowitz and Stegun ${ }^{9} 9.5 .10$ and 9.5.12). We estimate each factor of

$$
\left|J_{v}\left(j_{v, s_{0}}+z\right)\right|=\frac{\left|j_{v, s_{0}}+z\right|^{v}}{2^{v} \Gamma(v+1)} \prod_{s=1}^{\infty}\left|1-\left(\frac{j_{v, s_{0}}+z}{j_{v, s}}\right)^{2}\right|
$$

from below for $s_{*}<s_{0} \in \mathbb{N}$ and $z \in \mathbb{R}$ satisfying $|z| \leq\left(\inf _{s \in \mathbb{N}}\left(j_{v, s+1}-j_{v, s}\right) / 2\right) \wedge(\delta / 4)$. For $s=s_{0}$, we estimate as

$$
\left|1-\left(\frac{j_{v, s_{0}}+z}{j_{v, s_{0}}}\right)^{2}\right| \geq \frac{\left(2 j_{v, s_{0}}+z\right)|z|}{j_{v, s_{0}}^{2}} \geq \frac{|z|}{j_{v, s_{0}}} \geq \frac{c_{1}|z|}{s_{0}} .
$$

For $s_{0}<s \in \mathbb{N}$, we have

$$
1-\left(\frac{j_{v, s_{0}}+z}{j_{v, s}}\right)^{2} \geq 1-\left(\frac{j_{v, s_{0}}+z}{(s+v / 2-1 / 4) \pi-\delta}\right)^{2} \geq 1-\left(\frac{j_{v, s_{0}}+z}{\lfloor(s+v / 2-1 / 4) \pi-\delta\rfloor_{\left(\mathbb{Z}_{+}+1 / 2\right) \pi}}\right)^{2},
$$

where $\lfloor t\rfloor_{\left(\mathbb{Z}_{+}+1 / 2\right) \pi}=\max \left\{[0, t] \cap\left(\left(\mathbb{Z}_{+}+1 / 2\right) \pi\right)\right\}$ for any $t \geq 0$. For $s \in \mathbb{N} \cap\left[s_{*}, s_{0}\right)$, we have

$$
\left(\frac{j_{v, s_{0}}+z}{j_{v, s}}\right)^{2}-1 \geq\left(\frac{j_{v, s_{0}}+z}{(s+v / 2-1 / 4) \pi+\delta}\right)^{2}-1 \geq\left(\frac{j_{v, s_{0}}+z}{\lceil(s+v / 2-1 / 4) \pi+\delta\rceil_{\left(\mathbb{Z}_{+}+1 / 2\right) \pi}}\right)^{2}-1,
$$

where $\lceil t\rceil_{\left(\mathbb{Z}_{+}+1 / 2\right) \pi}=\min \left\{[t, \infty) \cap\left(\left(\mathbb{Z}_{+}+1 / 2\right) \pi\right)\right\}$ for any $t \geq 0$. We can also show

$$
\left\lfloor\left(s_{0}+s+v / 2-1 / 4\right) \pi-\delta\right\rfloor_{\left(\mathbb{Z}_{+}+1 / 2\right) \pi}=\left\lfloor\left(s_{0}+v / 2-1 / 4\right) \pi-\delta\right\rfloor_{\left(\mathbb{Z}_{+}+1 / 2\right) \pi}+s \pi
$$

for any $s \in \mathbb{N}$,

$$
\left\lceil\left(s_{0}-s+v / 2-1 / 4\right) \pi+\delta\right\rceil_{\left(\mathbb{Z}_{+}+1 / 2\right) \pi}=\left\lceil\left(s_{0}+v / 2-1 / 4\right) \pi+\delta\right\rceil_{\left(\mathbb{Z}_{+}+1 / 2\right) \pi}-s \pi
$$

for any $s_{0}-s_{*} \geq s \in \mathbb{N}$, and

$$
\begin{aligned}
& \left\lceil\left(s_{0}+v / 2-1 / 4\right) \pi+\delta\right\rceil_{\left(\mathbb{Z}_{+}+1 / 2\right) \pi}-\left\lfloor\left(s_{0}+v / 2-1 / 4\right) \pi-\delta\right\rfloor_{\left(\mathbb{Z}_{+}+1 / 2\right) \pi} \\
= & \left\{\begin{array}{l}
\pi \text { if } d\left(\left(s_{0}+v / 2-1 / 4\right) \pi,\left(\mathbb{Z}_{+}+1 / 2\right) \pi\right) \geq \delta \\
2 \pi \text { if } d\left(\left(s_{0}+v / 2-1 / 4\right) \pi,\left(\mathbb{Z}_{+}+1 / 2\right) \pi\right)<\delta .
\end{array}\right.
\end{aligned}
$$

If $d\left(\left(s_{0}+v / 2-1 / 4\right) \pi,\left(\mathbb{Z}_{+}+1 / 2\right) \pi\right)<\delta$, then we apply Eq. (A11) for $s_{0}+2 \leq s \in \mathbb{N}$ and apply Eq. (A12) for $s \in \mathbb{N} \cap\left[s_{*}, s_{0}-2\right]$. We apply

$$
1-\left(\frac{j_{v, s_{0}}+z}{j_{v, s_{0}+1}}\right)^{2} \geq \frac{\left(j_{v, s_{0}}+j_{v, s_{0}+1}+z\right)\left(j_{v, s_{0}}-j_{v, s_{0}+1}-z\right)}{j_{v, s_{0}+1}^{2}} \geq \frac{c_{2}}{s_{0}}
$$

for $s=s_{0}+1$ and

$$
\left(\frac{j_{v, s_{0}}+z}{j_{v, s_{0}-1}}\right)^{2}-1 \geq \frac{\left(j_{v, s_{0}}+j_{v, s_{0}-1}+z\right)\left(j_{v, s_{0}}-j_{v, s_{0}-1}+z\right)}{j_{v, s_{0}-1}^{2}} \geq \frac{c_{2}}{s_{0}}
$$

for $s=s_{0}-1$. Next, we use the following estimate; there exists a finite positive constant $c_{3}$ such that

$$
\prod_{m_{0} \neq m \in\left(\mathbb{Z}_{+}+1 / 2\right) \pi}\left|1-\left(\frac{w}{m}\right)^{2}\right| \geq c_{3} m_{0}
$$


for any $m_{0} \in\left(\mathbb{Z}_{+}+1 / 2\right) \pi$ and $w \in m_{0}+(\pi / 2)(-1,1)$. Indeed, because

$$
\prod_{m \in\left(\mathbb{Z}_{+}+1 / 2\right) \pi}\left\{1-\left(\frac{w}{m}\right)^{2}\right\}=\cos w
$$

(Abramowitz and Stegun ${ }^{9}$ 4.3.90), we have

$$
\begin{aligned}
& \prod_{m_{0} \neq m \in\left(\mathbb{Z}_{+}+1 / 2\right) \pi}\left|1-\left(\frac{w}{m}\right)^{2}\right|=\left|\frac{\cos w-\cos m_{0}}{1-\left(w / m_{0}\right)^{2}}\right| \\
= & \frac{m_{0}^{2}}{m_{0}+w}\left|\frac{\cos w-\cos m_{0}}{w-m_{0}}\right| \geq \frac{m_{0}}{3}\left|\int_{0}^{1} d t \sin \left((1-t) m_{0}+t w\right)\right| \\
= & \frac{m_{0}}{3} \int_{0}^{1} d t\left|\sin \left((1-t) m_{0}+t w\right)\right| \geq \frac{m_{0}}{3} \int_{0}^{1 / 2} d t\left|\sin \left((1-t) m_{0}+t w\right)\right| \\
\geq & \frac{m_{0}}{6} \min _{t \in m_{0^{+}}(\pi / 4)[-1,1]}|\sin t| .
\end{aligned}
$$

We take $m_{0}=\left\lceil\left(s_{0}-1+v / 2-1 / 4\right) \pi+\delta\right\rceil_{\left(\mathbb{Z}_{+}+1 / 2\right) \pi}=\left\lfloor\left(s_{0}+1+v / 2-1 / 4\right) \pi-\delta\right\rfloor_{\left(\mathbb{Z}_{+}+1 / 2\right) \pi}$. Then we have $j_{v, s_{0}}+z \in m_{0}+(\pi / 2)(-1,1)$ if $\delta \leq 2 \pi / 9$, as $|z| \leq \delta / 4$. Therefore,

$$
\begin{aligned}
& \left|J_{v}\left(j_{v, s_{0}}+z\right)\right| \\
\geq & \frac{c_{4}\left|z \| j_{v, s_{0}}+z\right|^{v}}{s_{0}^{2}} \prod_{s=1}^{s_{*}-1}\left|1-\left(\frac{j_{v, s_{0}}+z}{j_{v, s}}\right)^{2}\right| \\
& \left.\times \prod_{\left\lceil\left(s_{*}+v / 2-1 / 4\right) \pi+\delta\right\rceil_{\left(\mathbb{Z}_{+}+1 / 2\right) \pi}>m \in\left(\mathbb{Z}_{+}+1 / 2\right) \pi}\left|1-\left(\frac{j_{v, s_{0}}+z}{m}\right)^{2}\right|\right)^{-1} \\
\geq & c_{5}|z| / s_{0}^{c_{6}} .
\end{aligned}
$$

If $d\left(\left(s_{0}+v / 2-1 / 4\right) \pi,\left(\mathbb{Z}_{+}+1 / 2\right) \pi\right) \geq \delta$, then $d\left(j_{v, s_{0}},\left(\mathbb{Z}_{+}+1 / 2\right) \pi\right) \geq \delta / 2$ and $d\left(j_{v, s_{0}}+z,\left(\mathbb{Z}_{+}\right.\right.$ $+1 / 2) \pi) \geq \delta / 4$ as $|z| \leq \delta / 4$. Then we apply Eqs. (A11) and (A12) for $s_{0}+1 \leq s \in \mathbb{N}$ and $s \in$ $\mathbb{N} \cap\left[s_{*}, s_{0}-1\right]$, respectively, and use Eq. (A19). Then we have

$$
\begin{aligned}
& \left|J_{v}\left(j_{v, s_{0}}+z\right)\right| \\
\geq & c_{7} \frac{|z|}{s_{0}}\left|j_{v, s_{0}}+z\right|^{v}\left|\cos \left(j_{v, s_{0}}+z\right)\right| \prod_{s=1}^{s_{*}-1}\left|1-\left(\frac{j_{v, s_{0}}+z}{j_{v, s}}\right)^{2}\right| \\
& \left.\times \prod_{\left\lceil\left(s_{*}+v / 2-1 / 4\right) \pi+\delta\right\rceil_{\left(\mathbb{Z}_{+}+1 / 2\right) \pi}>m \in\left(\mathbb{Z}_{+}+1 / 2\right) \pi}\left|1-\left(\frac{j_{v, s_{0}}+z}{m}\right)^{2}\right|\right)^{-1} \\
\geq & c_{8}|z| / s_{0}^{c_{9}} .
\end{aligned}
$$

Therefore, we have shown

$$
\left|J_{v}\left(j_{v, s}+z\right)\right|\left|j_{v, s}+z\right|^{c_{10}} \geq c_{11}|z|
$$

for $s_{*}<s \in \mathbb{N}$ and $z \in \mathbb{R}$ satisfying $|z| \leq\left(\inf _{s \in \mathbb{N}}\left(j_{v, s+1}-j_{v, s}\right) / 2\right) \wedge(\delta / 4)=: \zeta$. By the same asymptotic behavior as in Eq. (A8), there exists $s_{* *} \in \mathbb{N}$ such that

$$
\left|j_{v, s}-\left(s+\frac{v}{2}-\frac{1}{4}\right) \pi\right| \leq \frac{\zeta}{2}
$$

for $s_{* *}<s \in \mathbb{N}$. We also recall that

$$
J_{v}(t)=\sqrt{\frac{2}{\pi t}}\left\{\cos \left(t-\frac{2 v+1}{4} \pi\right)+O\left(\frac{1}{t}\right)\right\}
$$

(cf. Abramowitz and Stegun ${ }^{9}$ 9.2.1). Then, we establish the existence of $R_{*} \in(0, \infty)$ such that

$$
\inf \left\{\left|J_{v}(t)\right| \sqrt{|t|}: t \in\left[R_{*}, \infty\right) \backslash \bigcup_{s \in \mathbb{N}}\left(j_{v, s}+(-\zeta, \zeta)\right)\right\}>0 .
$$

Therefore, by changing the constants, we can complete the proof of this proposition. 


\section{APPENDIX B: BEHAVIOR OF FUNCTION $\sigma$ AT $\partial$ supp $\sigma$}

In this section, we prove that the function $\sigma$ in Example 1.1 (i) satisfies condition (A2). We consider only (iv) and (v) as the other conditions are easily checked. The fundamental lemma is the following:

Lemma B.1.

$$
\sqrt{T(D+T)} \leq \mathcal{H}^{1}(S(D, T)) \leq 2 \sqrt{T(D+T)}
$$

for any $D, T \geq 0$, where

$$
S(D, T):=\left\{\left(x_{1}, x_{2}\right) \in[D, D+T] \times[0, \infty): x_{1}^{2}+x_{2}^{2}=(D+T)^{2}\right\} .
$$

Then, we can easily prove that condition (A2) (iv) holds as $\operatorname{supp} \sigma$ is an ellipse because the curvature does not vanish. We have

$$
\begin{aligned}
&\{x \in \operatorname{supp} \sigma: d(x, y)=s+d(y, \operatorname{supp} \sigma)\} \\
& \subset\left\{x \in \mathbb{R}^{2}:|y-x|=\left|y-y_{\sigma}\right|+s,(y-x) \cdot\left(y-y_{\sigma}\right) \geq\left|y-y_{\sigma}\right|^{2}\right\}
\end{aligned}
$$

and

$$
\mathcal{H}^{1}(\{x \in \operatorname{supp} \sigma: d(x, y)=s+d(y, \operatorname{supp} \sigma)\}) \leq 2 \mathcal{H}^{1}\left(S\left(\left|y-y_{\sigma}\right|, s\right)\right) \leq c s^{1 / 2},
$$

for any $y \in \mathbb{R}^{2} \backslash \overline{\operatorname{supp} \sigma}$, where $y_{\sigma} \in \partial \operatorname{supp} \sigma$ is chosen so that $\left|y-y_{\sigma}\right|=d(y, \operatorname{supp} \sigma)$. The uniqueness of $y_{\sigma}$ is due to the convexity of the ellipse.

To treat condition (A2) (v), we dominate the function $\sigma$ from below on the set

$\mathcal{S}_{\sigma}(y, s, \delta):=\left\{x \in \mathbb{R}^{2}:|y-x|=\left|y-y_{\sigma}\right|+s,(y-x) \cdot\left(y-y_{\sigma}\right) \geq\left(\left|y-y_{\sigma}\right|+s(1-\delta)\right)\left|y-y_{\sigma}\right|\right\}$

with some $\delta \in(0,1)$. Any point of this set is represented as

$$
y_{\sigma}-s\left(1-\delta^{\prime}\right) \frac{y-y_{\sigma}}{\left|y-y_{\sigma}\right|} \pm \sqrt{\left(s\left(2-\delta^{\prime}\right)+2\left|y-y_{\sigma}\right|\right) s \delta^{\prime}} v=: x\left( \pm, s, \delta^{\prime}\right),
$$

where $\delta^{\prime} \in[0, \delta]$ and $v$ is one of the unit vectors perpendicular with $y-y_{\sigma}$. We set $\mathcal{S}_{\sigma, \pm}(y, s, \delta):=$ $\left\{x\left( \pm, s, \delta^{\prime}\right): \delta \in[0, \delta]\right\}$. We may assume that $\sigma$ is represented as Eq. (A1) with $a_{1} \geq a_{2}>0$. Then, we have

$$
\begin{aligned}
& \sigma^{1 / v}\left(x\left( \pm, s, \delta^{\prime}\right)\right)=\bar{\sigma}^{2}-\sum_{j=1}^{2} a_{j}^{2}\left(y_{\sigma, j}-s\left(1-\delta^{\prime}\right) \frac{y_{j}-y_{\sigma, j}}{\left|y-y_{\sigma}\right|} \pm \sqrt{\left(s\left(2-\delta^{\prime}\right)+2\left|y-y_{\sigma}\right|\right) s \delta^{\prime}} v_{j}\right)^{2} \\
= & -\sum_{j=1}^{2} a_{j}^{2}\left(s\left(1-\delta^{\prime}\right) \frac{y_{j}-y_{\sigma, j}}{\left|y-y_{\sigma}\right|} \mp \sqrt{\left(s\left(2-\delta^{\prime}\right)+2\left|y-y_{\sigma}\right|\right) s \delta^{\prime}} v_{j}\right)^{2} \\
& +2 \sum_{j=1}^{2} a_{j}^{2} y_{\sigma, j}\left(s\left(1-\delta^{\prime}\right) \frac{y_{j}-y_{\sigma, j}}{\left|y-y_{\sigma}\right|} \mp \sqrt{\left(s\left(2-\delta^{\prime}\right)+2\left|y-y_{\sigma}\right|\right) s \delta^{\prime}} v_{j}\right) \\
\geq & -a_{1}^{2}\left(s^{2}\left(1-\delta^{\prime}\right)^{2}+\left(s\left(2-\delta^{\prime}\right)+2\left|y-y_{\sigma}\right|\right) s \delta^{\prime}\right) \\
& \left.+2 \sum_{j=1}^{2} a_{j}^{2} y_{\sigma, j} s(1-\delta) \frac{y_{j}-y_{\sigma, j}}{\left|y-y_{\sigma}\right|} \mp 2 \sqrt{\left(s\left(2-\delta^{\prime}\right)+2\left|y-y_{\sigma}\right|\right) s \delta^{\prime}} \sum_{j=1}^{2} a_{j}^{2} y_{\sigma, j} v_{j}\right) .
\end{aligned}
$$

Thus, for an $\epsilon \in\{+,-\}$, depending only on $y$ and $y_{\sigma}$, we have

$$
\sigma^{1 / v}\left(x\left(\epsilon, s, \delta^{\prime}\right)\right) \geq-a_{1}^{2}\left(s^{2}+2\left|y-y_{\sigma}\right| s \delta\right)+2 a_{2}^{2} s(1-\delta) y_{\sigma} \cdot \frac{y-y_{\sigma}}{\left|y-y_{\sigma}\right|} .
$$

We can show that

$$
\frac{y_{\sigma}}{\left|y_{\sigma}\right|} \cdot \frac{y-y_{\sigma}}{\left|y-y_{\sigma}\right|} \geq \frac{2 a_{1} a_{2}}{a_{1}^{2}+a_{2}^{2}}
$$


Indeed, if we set $y_{\sigma}=\left(b_{1} \cos \theta, b_{2} \sin \theta\right)$, where $b_{j}=\bar{\sigma} / a_{j}$, then an outward normal vector $\left(b_{2} \cos \theta\right.$, $\left.b_{1} \sin \theta\right)$ of $\partial \operatorname{supp} \sigma$ at $y_{\sigma}$ is parallel with $y-y_{\sigma}$,

$$
\frac{y-y_{\sigma}}{\left|y-y_{\sigma}\right|}=\left(b_{1}^{2} \sin ^{2} \theta+b_{2}^{2} \cos ^{2} \theta\right)^{-1 / 2}\left(\begin{array}{c}
b_{2} \cos \theta \\
b_{1} \sin \theta
\end{array}\right)
$$

and

$$
\frac{y_{\sigma}}{\left|y_{\sigma}\right|} \cdot \frac{y-y_{\sigma}}{\left|y-y_{\sigma}\right|}=\left\{\left(b_{1}^{2} \sin ^{2} \theta+b_{2}^{2} \cos ^{2} \theta\right)\left(b_{1}^{2} \cos ^{2} \theta+b_{2}^{2} \sin ^{2} \theta\right)\right\}^{-1 / 2} b_{1} b_{2} \geq \frac{2 b_{1} b_{2}}{b_{1}^{2}+b_{2}^{2}}=\frac{2 a_{1} a_{2}}{a_{1}^{2}+a_{2}^{2}} .
$$

Thus, we have

$$
\sigma^{1 / v}\left(x\left( \pm, s, \delta^{\prime}\right)\right) \geq \frac{2(1-\delta) \bar{\sigma} a_{1} a_{2}^{2} s}{a_{1}^{2}+a_{2}^{2}}
$$

if $s \leq(1-\delta) \bar{\sigma} a_{2}^{2} /\left(a_{1}\left(a_{1}^{2}+a_{2}^{2}\right)\right)$ and $\left|y-y_{\sigma}\right| \leq(1-\delta) \bar{\sigma} a_{2}^{2} /\left(2 \delta a_{1}\left(a_{1}^{2}+a_{2}^{2}\right)\right)$. Therefore, for $y \in$ $\Sigma\left(0,(1-\delta) \bar{\sigma} a_{2}^{2} /\left(2 \delta a_{1}\left(a_{1}^{2}+a_{2}^{2}\right)\right), \pi\right)$ and $s \in\left[0,(1-\delta) \bar{\sigma} a_{2}^{2} /\left(a_{1}\left(a_{1}^{2}+a_{2}^{2}\right)\right)\right]$, we have

$$
\left\{x \in \mathbb{R}^{2}: \sigma(x) \geq\left\{\frac{2(1-\delta) \bar{\sigma} a_{1} a_{2}^{2} s}{a_{1}^{2}+a_{2}^{2}}\right\}^{v}, d(x, y)=s+d(y, \operatorname{supp} \sigma)\right\} \supset \mathcal{S}_{\sigma, \epsilon}(y, s, \delta)
$$

and

$$
\begin{aligned}
& \mathcal{H}^{1}\left(\left\{x \in \mathbb{R}^{2}: \sigma(x) \geq\left\{\frac{2(1-\delta) \bar{\sigma} a_{1} a_{2}^{2} s}{a_{1}^{2}+a_{2}^{2}}\right\}^{v}, d(x, y)=s+d(y, \operatorname{supp} \sigma)\right\}\right) \\
\geq & \mathcal{H}^{1}(S(d(y, \operatorname{supp} \sigma)+s(1-\delta), s \delta) \geq \sqrt{s \delta(d(y, \operatorname{supp} \sigma)+s)},
\end{aligned}
$$

which implies condition (A2) (v).

\footnotetext{
${ }^{1}$ Carmona, R. and Lacroix, J., Spectral Theory of Random Schrödinger Operators (Birkhäuser Boston, Inc., Boston, MA, 1990).

${ }^{2}$ Doi, S., Iwatsuka, A., and Mine, T., Math. Z. 237, 335-371 (2001).

${ }^{3}$ Erdélyi, A., Magnus, W., Oberhettinger, F., and Tricomi, F. G., Tables of Integral Transforms. Vol. I: Based, in Part, on Notes Left by Harry Bateman (McGraw-Hill Book Company, Inc., New York, Toronto, London, 1954).

${ }^{4}$ Erdős, L. and Hasler, D., Commun. Math. Phys. 309, 507-542 (2012).

${ }^{5}$ Erdős, L. and Hasler, D., Ann. Henri Poincaré 13, 1719-1731 (2012).

${ }^{6}$ Erdős, L. and Hasler, D., J. Stat. Phys. 146, 900-923 (2012).

${ }^{7}$ Frőhlich, J. and Spencer, T., Commun. Math. Phys. 88, 151-184 (1983).

${ }^{8}$ Germinet, F. and Klein, A., Commun. Math. Phys. 222, 415-448 (2001).

${ }^{9}$ Handbook of Mathematical Functions with Formulas, Graphs, And Mathematical Tables, edited by M. Abramowitz and I. A. Stegun (Dover Publications, Inc., New York, 1992).

${ }^{10}$ Kirsch, W. and Martinelli, F., Commun. Math. Phys. 89, 27-40 (1983).

${ }^{11}$ Leinfelder, H. and Simader, C. G., Math. Z. 176, 1-19 (1981).

${ }^{12}$ Nakamura, S., Commun. Math. Phys. 214, 565-572 (2000).

${ }^{13}$ Nualart, D., The Malliavin Calculus and Related Topics (Springer, New York, 2010).

${ }^{14}$ Pastur, L. and Figotin, A., Spectra of Random and Almost-periodic Operators (Springer-Verlag, Berlin, 1992).

${ }^{15}$ Reed, M. and Simon, B., Methods of Modern Mathematical Physics. IV. Analysis of Operators (Academic Press [Harcourt Brace Jovanovich Publishers], New York, 1978).

${ }^{16}$ Shigekawa, I., Stochastic Analysis (American Mathematical Society, Providence, RI, 2004).

${ }^{17}$ Ueki, N., Osaka J. Math. 31, 177-187 (1994).

18 Ueki, N., Ann. Henri Poincare 1, 473-498 (2000).

${ }^{19}$ Ueki, N., J. Funct. Anal. 263, 2024-2050 (2012).

${ }^{20}$ Wegner, F., Z. Phys. B 44, 9-15 (1981).
} 